University of Nebraska - Lincoln

DigitalCommons@University of Nebraska - Lincoln

\title{
Accumulation of metals in fish from lead-zinc mining areas of southeastern Missouri, USA
}

Christopher J. Schmitt

U.S. Geological Survey, cjschmitt@usgs.gov

William G. Brumbaugh

United States Geological Survey, bbrumbaugh@usgs.gov

Thomas W. May

U.S. Geological Survey, tmay@usgs.gov

Follow this and additional works at: https://digitalcommons.unl.edu/usgsstaffpub

Schmitt, Christopher J.; Brumbaugh, William G.; and May, Thomas W., "Accumulation of metals in fish from lead-zinc mining areas of southeastern Missouri, USA" (2007). USGS Staff -- Published Research. 564.

https://digitalcommons.unl.edu/usgsstaffpub/564

This Article is brought to you for free and open access by the US Geological Survey at DigitalCommons@University of Nebraska - Lincoln. It has been accepted for inclusion in USGS Staff -- Published Research by an authorized administrator of DigitalCommons@University of Nebraska - Lincoln. 


\title{
Accumulation of metals in fish from lead-zinc mining areas of southeastern Missouri, USA
}

\author{
Christopher J. Schmitt*, William G. Brumbaugh, Thomas W. May \\ US Geological Survey (USGS), Columbia Environmental Research Center (CERC), 4200 New Haven Road, Columbia, MO 65201, USA
}

Received 19 April 2006; received in revised form 30 October 2006; accepted 3 November 2006

Available online 15 February 2007

\begin{abstract}
The potential effects of proposed lead-zinc mining in an ecologically sensitive area were assessed by studying a nearby mining district that has been exploited for about $30 \mathrm{yr}$ under contemporary environmental regulations and with modern technology. Blood and liver samples representing fish of three species (largescale stoneroller, Campostoma oligolepis, $n=91$; longear sunfish, Lepomis megalotis, $n=105$; and northern hog sucker, Hypentelium nigricans, $n=20$ ) were collected from 16 sites representing a range of conditions relative to lead-zinc mining and ore beneficiation in southeastern Missouri. Samples were analyzed for lead, zinc, and cadmium, and for a suite of biomarkers (reported in a companion paper). A subset of the hog sucker $(n=9)$ representing three sites were also analyzed for nickel and cobalt. Blood and liver lead concentrations were highly correlated $(r=0.84-0.85, P<0.01)$ in all three species and were significantly (ANOVA, $P<0.01)$ greater at sites $<10 \mathrm{~km}$ downstream of active lead-zinc mines and mills and in a historical lead-zinc mining area than at reference sites, including a site in the area proposed for new mining. Correlations between blood and liver cadmium concentrations were less evident than for lead but were nevertheless statistically significant $(r=0.26-0.69, P<0.01-0.07)$. Although blood and liver cadmium concentrations were highest in all three species at sites near mines, within-site variability was greater and mining-related trends were less evident than for lead. Blood and liver zinc concentrations were significantly correlated only in stoneroller $(r=0.46, P<0.01)$ and mining-related trends were not evident. Concentrations of cobalt and nickel in blood and liver were significantly higher (ANOVA, $P<0.01$ ) at a site near an active mine than at a reference site and a site in the historical lead-zinc mining area. These findings confirm previous studies indicating that lead and other metals are released to streams from active lead-zinc mines and are available for uptake by aquatic organisms.
\end{abstract}

(C) 2006 Elsevier Inc. All rights reserved.

Keywords: Metals; Mining; Lead; Cadmium; Zinc; Cobalt; Nickel; Stoneroller; Longear sunfish; Hog sucker

\section{Introduction}

The extensive lead $(\mathrm{Pb})$ deposits of southern Missouri were first discovered by early French explorers of the Mississippi River valley. Subsequently discovered ores containing variable amounts of $\mathrm{Pb}$, zinc $(\mathrm{Zn})$, copper $(\mathrm{Cu})$, cadmium $(\mathrm{Cd})$, cobalt $(\mathrm{Co})$, nickel $(\mathrm{Ni})$, silver, and other metals have been exploited at varying levels of intensity for more than $300 \mathrm{yr}$. The most productive historical deposits were those of the Old Lead Belt, in southeastern Missouri, and the Tri-States Mining District, in southwestern

\footnotetext{
*Corresponding author. Fax: + 15738761896.

E-mail address: cjschmitt@usgs.gov (C.J. Schmitt).
}

Missouri, southeastern Kansas, and northeastern Oklahoma. The mines and related facilities in these districts were closed by the 1970s, but they left behind a legacy of contaminated land and water resources owing to both the relative inefficiency of the extraction technologies available during the active mining period and the fact that most early mining pre-dated environmental regulation. Elevated concentrations of metals in water, sediment, and biota and effects on aquatic organisms in these historical mining areas are well documented (e.g., Brumbaugh et al., 2005; Dwyer et al., 1988; Gale et al., 2004; Schmitt et al., 1984, 1992, 1993, 2005; Wildhaber et al., 2000).

The discovery in the 1950s of the Viburnum Trend, a geologic formation containing significant $\mathrm{Pb}-\mathrm{Zn}$ reserves, 
led to the development of the New Lead Belt (NLB) mining district in southeastern Missouri (Fig. 1). In contrast to previous large-scale mining, the mines and mills of the NLB were developed and have operated under contemporary environmental regulations and with the most efficient extraction and treatment technologies available to the industry (Wixson and Jennett, 1975; Wixson, 1978). Elevated metals concentrations and biochemical effects in fish from NLB streams have nevertheless been reported from a limited number of sites (e.g., Schmitt et al., 1993, 2002).

By the 1980s, two decades of mining in the NLB had depleted the Viburnum Trend and mining activity declined; however, additional potentially exploitable deposits were discovered further to the southwest. The exploration area lies within the boundary of the Mark Twain National Forest, in an environmentally sensitive part of Missouri that hosts recreationally and ecologically significant resources such as springs, caves, a National Park, and a

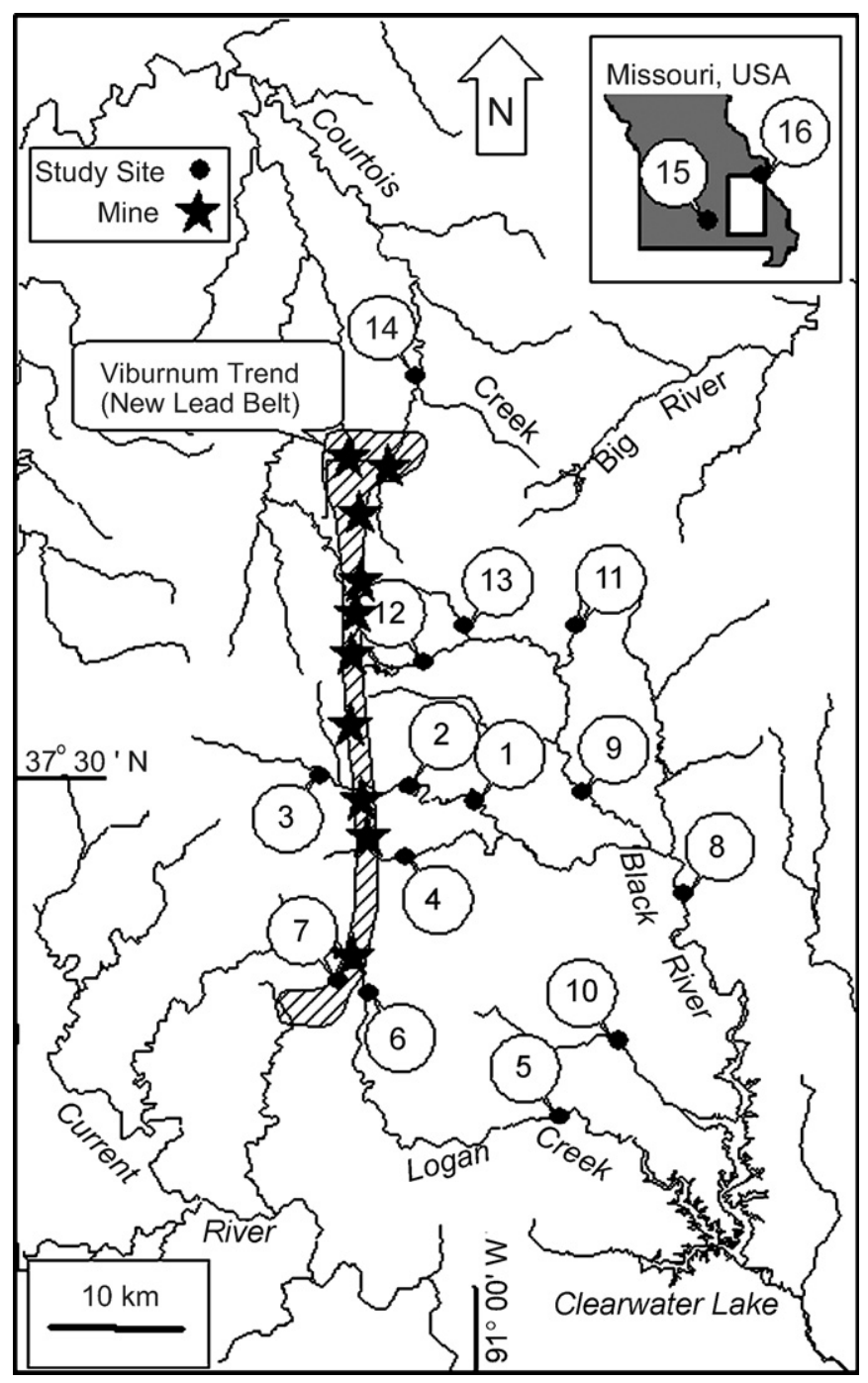

Fig. 1. Map of the southeastern Missouri study area showing the locations of study sites (1-16) and New Lead Belt mines along the Viburnum Trend.
Federally designated Scenic River, all of which could be threatened by mining. In response to public concerns, a multi-year interdisciplinary investigation was initiated to evaluate potential environmental effects of expanded mining in the exploration area (Imes, 2002). The study described here, which represents part of the larger investigation, was focused in the NLB as a model for the potential consequences of expanded mining elsewhere in southern Missouri.

Our primary objective was to document metals concentrations and biomarkers of metals exposure in fish from streams representing a wide range of conditions related to contemporary $\mathrm{Pb}-\mathrm{Zn}$ mining in southeastern Missouri. This was achieved by collecting blood and liver samples of fish from the exploration area, other reference areas, and sites influenced to varying degrees by active and historical mining; analyzing the samples for metals and biomarkers of metals exposure; and comparing the results to pertinent data from the scientific literature. Secondary objectives included the refinement of biomarker methods for use with small fish and the documentation of metals concentrations and biomarker responses in a species for which they had not been evaluated previously. The use of small fish was necessary because many NLB mines and their associated mills and tailings disposal facilities are located near headwater streams that are too small to support large fish in the numbers necessary for metals and biomarker analyses by traditional methods (e.g., Schmitt et al., 1984, 1993). Results of the metals analyses are presented here. Biomarker results are presented in a companion paper (Schmitt et al., 2007).

\section{Methods of study}

\subsection{Study design}

Fish of two species, largescale stoneroller (Campostoma oligolepis; henceforth stoneroller) and longear sunfish (Lepomis megalotis; sunfish), were collected from each site. Northern hog sucker (Hypentelium nigricans; hog sucker), a species collected in previous studies (Schmitt et al., 1984, 1993), were retained for analysis when captured incidentally to facilitate temporal and inter-species comparisons. Sites 1-14, in the Black and Meramec River systems, were selected to represent the range of conditions present in the NLB relative to mining. These included reference sites $(n=3)$, which were located upstream of all mining activity; and sites situated $1.4-49.8 \mathrm{~km}$ downstream of NLB mines and ore processing facilities (Fig. 1; Table 1). Sunfish were not present in the immediate vicinity of Sites 6 and 14 but were obtained $2-5 \mathrm{~km}$ downstream from the locations identified in Table 1. A site on the Eleven Point River (Site 15) was sampled to represent existing conditions in the exploration area and also served as an additional reference site. The Big River (Site 16), which has been contaminated by mine tailings from historical mining in the Old Lead Belt (e.g., Gale et al., 2004; Schmitt et al., 1984), was also sampled. Sites 2, 5, 9, 14, and 16 (Table 1) were also sampled for blood metals and biomarkers by previous studies (Dwyer et al., 1988; Schmitt et al., 1984, 1993, 2005) and were used for temporal and inter-species comparisons. All sites were sampled in September 2001 except Site 16, which was sampled in early December 2001 (Table 1). Concentrations of $\mathrm{Pb}, \mathrm{Zn}$, and $\mathrm{Cd}$ were measured in the blood and liver of all fish. During the conduct of our study, a related investigation indicated the presence of elevated concentrations of $\mathrm{Co}$ and $\mathrm{Ni}$ in sediments at some NLB sites (Besser 
Table 1

Fish collection sites in southeastern Missouri

\begin{tabular}{|c|c|c|c|c|c|}
\hline $\begin{array}{l}\text { Site no. and } \\
\text { (type) }^{\mathrm{a}}\end{array}$ & Stream and location & County & Latitude, longitude ${ }^{\mathrm{b}}$ & $\begin{array}{l}\text { Sampling } \\
\text { date(s) }\end{array}$ & $\begin{array}{l}\text { Metals source(s) and distance(s) } \\
\text { upstream }(\mathrm{km})\end{array}$ \\
\hline 1 (D) & West Fork Black River at Sutton Bluff & Reynolds & $37^{\circ} 28^{\prime} 33.6^{\prime \prime} \mathrm{N}, 91^{\circ} 00^{\prime} 32.4^{\prime \prime} \mathrm{W}$ & 09-10-01 & $\begin{array}{l}\text { West Fork mine and mill (16.5); } \\
\text { Brushy Creek mine and mill (24.6) }\end{array}$ \\
\hline $2(\mathrm{M})$ & West Fork Black River at West Fork & Reynolds & $37^{\circ} 29^{\prime} 49.2^{\prime \prime} \mathrm{N}, 91^{\circ} 05^{\prime} 13.2^{\prime \prime} \mathrm{W}$ & 09-11-01 & $\begin{array}{l}\text { West Fork mine and mill (2.2); Brushy } \\
\text { Creek mine and mill (10.3) }\end{array}$ \\
\hline $3(\mathrm{R})$ & West Fork Black River near Greeley & Reynolds & $37^{\circ} 30^{\prime} 39.6^{\prime \prime} \mathrm{N}, 91^{\circ} 09^{\prime} 43.2^{\prime \prime} \mathrm{W}$ & $\begin{array}{l}09-12-01 \\
09-26-01\end{array}$ & None (reference site) \\
\hline $4(\mathrm{M})$ & Bee Fork Black River near Bee Fork Church & Reynolds & $37^{\circ} 26^{\prime} 31.2^{\prime \prime} \mathrm{N}, 91^{\circ} 04^{\prime} 58.8^{\prime \prime} \mathrm{W}$ & $\begin{array}{l}09-14-01 \\
09-26-01\end{array}$ & Fletcher mine and mill (1.4) \\
\hline 5 (D) & Logan Creek at Ellington & Reynolds & $37^{\circ} 14^{\prime} 49.2^{\prime \prime} \mathrm{N}, 91^{\circ} 58^{\prime} 01.2^{\prime \prime} \mathrm{W}$ & 09-12-01 & Sweetwater mine and mill (31.3-34.1) \\
\hline $6(\mathrm{M})$ & Logan Creek near Corridon & Reynolds & $37^{\circ} 20^{\prime} 24.0^{\prime \prime} \mathrm{N}, 91^{\circ} 07^{\prime} 12.0^{\prime \prime} \mathrm{W}$ & 09-13-01 & Sweetwater mine and mill (4.4) \\
\hline 7 (M) & Sweetwater Creek near Corridon & Reynolds & $37^{\circ} 19^{\prime} 48.0^{\prime \prime} \mathrm{N}, 91^{\circ} 08^{\prime} 09.6^{\prime \prime} \mathrm{W}$ & 09-13-01 & Sweetwater mine and mill (2.6) \\
\hline 8 (D) & Black River near Lesterville & Reynolds & $37^{\circ} 25^{\prime} 01.2^{\prime \prime} \mathrm{N}, 90^{\circ} 49^{\prime} 30.0^{\prime \prime} \mathrm{W}$ & 09-24-01 & $\begin{array}{l}\text { New Lead Belt mines and mills } \\
(35.7-49.8)\end{array}$ \\
\hline 9 (D) & Middle Fork Black River at Black & Reynolds & $37^{\circ} 31^{\prime} 26.4^{\prime \prime} \mathrm{N}, 90^{\circ} 56^{\prime} 06.0^{\prime \prime} \mathrm{W}$ & 09-24-01 & $\begin{array}{l}\text { Magmont mine and mill (29.0); Buick } \\
\text { mine and mill (22.0) }\end{array}$ \\
\hline $10(\mathrm{R})$ & Sinking Creek near Redford & Reynolds & $37^{\circ} 18^{\prime} 36.0^{\prime \prime} \mathrm{N}, 90^{\circ} 52^{\prime} 40.8^{\prime \prime} \mathrm{W}$ & 09-25-01 & None (reference site) \\
\hline $11(\mathrm{R})$ & Middle Fork Black River near Redmondville & Iron & $37^{\circ} 37^{\prime} 01.2^{\prime \prime} \mathrm{N}, 90^{\circ} 58^{\prime} 01.2^{\prime \prime} \mathrm{W}$ & $\begin{array}{l}09-25-01 \\
09-26-01\end{array}$ & None (reference site) \\
\hline $12(\mathrm{M})$ & Strother Creek near Oates & Iron & $37^{\circ} 36^{\prime} 07.2^{\prime \prime} \mathrm{N}, 91^{\circ} 01^{\prime} 40.8^{\prime \prime} \mathrm{W}$ & $09-25-01$ & Buick mine and mill (3.9) \\
\hline 13 (D) & Neals Creek near Oates & Iron & $37^{\circ} 36^{\prime} 28.8^{\prime \prime} \mathrm{N}, 91^{\circ} 01^{\prime} 01.2^{\prime \prime} \mathrm{W}$ & 09-26-01 & Magmont mine and mill (10.9) \\
\hline $14(\mathrm{M})$ & Courtois Creek at Courtois & Washington & $37^{\circ} 46^{\prime} 04.8^{\prime \prime} \mathrm{N}, 91^{\circ} 04^{\prime} 15.6^{\prime \prime} \mathrm{W}$ & 09-27-01 & Viburnum mines and mills (4.9-7.6) \\
\hline 15 (R/EA) & Eleven Point River at Cane Bluff & Oregon & $36^{\circ} 47^{\prime} 45.6^{\prime \prime} \mathrm{N}, 91^{\circ} 24^{\prime} 18.0^{\prime \prime} \mathrm{W}$ & $\begin{array}{l}09-27-01 \\
09-28-01\end{array}$ & None (exploration area; reference site) \\
\hline 16 (BR) & Big River at St. Francois State Park & St. Francois & $37^{\circ} 57^{\prime} 21.6^{\prime \prime} \mathrm{N}, 90^{\circ} 32^{\prime} 31.2^{\prime \prime} \mathrm{W}$ & 12-07-01 & Old Lead Belt mines (22.1-30.2) \\
\hline
\end{tabular}

${ }^{\mathrm{a}} \mathrm{M},<10 \mathrm{~km}$ downstream of New Lead Belt mine; D, > $10 \mathrm{~km}$ downstream; R, upstream of all mining (reference); EA, exploration area; BR, Big River.

${ }^{\mathrm{b}}$ As determined by global positioning system $(\mathrm{GPS})$ receiver $( \pm 10 \mathrm{~m})$; datum $=$ World Geodetic System $($ WGS $) 84$.

et al., 2003). Blood and liver samples representing hog sucker from three sites were therefore re-analyzed for $\mathrm{Ni}$ and $\mathrm{Co}$ to determine whether these metals had also accumulated in fish.

\subsection{Field procedures}

Most fish were collected by DC electrofishing and by seining. Sunfish were also collected by hook-and-line. The collection target was six (each) adult stoneroller (75-150 mm total length, 3-20 g) and juvenile sunfish $(100-150 \mathrm{~mm}, 30-60 \mathrm{~g})$ samples at each site. Hog sucker were larger $(165-375 \mathrm{~mm}, 40-650 \mathrm{~g})$. Most fish were analyzed individually; however, because the sunfish and stoneroller at several sites were small, some were analyzed as composites, with a "sample" representing the number of fish (typically 1-3) necessary to provide approximately $400 \mu \mathrm{L}$ of blood. Fish were held alive in plastic containers filled with aerated stream water for $\leqslant 2 \mathrm{~h}$ following capture.

Blood was collected from stoneroller and sunfish with a chilled $\left(0{ }^{\circ} \mathrm{C}\right)$, heparinized microcapillary tube $(75 \mu \mathrm{L}$ for stoneroller and small sunfish, $370 \mu \mathrm{L}$ for larger sunfish) after severing the caudal peduncle with a razor blade. Blood was obtained from hog sucker by caudal veinipuncture using a heparinized $(6 \mathrm{IU} / \mathrm{mL})$ needle and syringe. The blood was dispensed immediately into a chilled $2-\mathrm{mL}$ cryogenic tube from which $200 \mu \mathrm{L}$ for metals analysis was transferred using a heparinized microcapillary tube to a pre-weighed, acid-cleaned, $10-\mathrm{mL}$ borosilicate glass tube with a Teflon ${ }^{\circledR}$ cap, which was then frozen immediately in dry ice. The remainder was retained for biomarker analyses (Schmitt et al., 2007). Following blood collection the fish was euthanized by cervical dislocation, measured (total length, $\mathrm{mm}$ ), and weighed $(\mathrm{g})$. The abdominal cavity was opened by dissection and a liver sample (ca. $0.5 \mathrm{~g}$ ) was obtained and divided into three approximately equal sub-samples for metals and biomarker analyses (Schmitt et al., 2007). The gender of the fish was determined by observation of the gonads. The metals samples were placed in acidcleaned $0.5-\mathrm{mL}$ cryogenic vials and frozen immediately in dry ice. All contact surfaces and dissecting instruments were thoroughly cleaned with laboratory detergent, de-ionized $\mathrm{H}_{2} \mathrm{O}$, and acetone between samples.
Upon return from the field the samples were stored frozen at $-20{ }^{\circ} \mathrm{C}$ until analyzed.

\subsection{Laboratory methods}

Frozen blood and liver samples were freeze-dried, digested with $\mathrm{HNO}_{3}$ and $\mathrm{H}_{2} \mathrm{O}_{2}$, and analyzed for $\mathrm{Pb}, \mathrm{Cd}$, and $\mathrm{Zn}$ by inductively coupled plasma mass spectrometry (ICPMS) as described by Brumbaugh et al. (2005). Blood moisture content was determined from weight loss during lyophilization; liver moisture content was not determined. Method limits-of-detection (LODs), calculated independently for each sample assuming a digestion weight of $50 \mathrm{mg}$ of dry blood or liver, were $0.1-4.0 \mu \mathrm{g} / \mathrm{g}$ dry weight $(\mathrm{dw})$ for $\mathrm{Zn}, 0.003-0.007 \mu \mathrm{g} / \mathrm{g} \mathrm{dw}$ for $\mathrm{Cd}$, and $0.005-0.07 \mu \mathrm{g} / \mathrm{g}$ for $\mathrm{Pb}$. Blood and liver samples from nine hog sucker representing Sites 11, 12, and 16 were also analyzed by ICPMS for Co and $\mathrm{Ni}$; method LODs were $0.003 \mu \mathrm{g} / \mathrm{g} \mathrm{dw}$ for Co and $0.02 \mu \mathrm{g} / \mathrm{g} \mathrm{dw}$ for Ni. All metals results were analyzed and reported as dw concentrations.

\subsection{Data set composition and statistical analyses}

Release 9.1 of the Statistical Analysis System (SAS Institute, Cary, NC) was used for all statistical analyses. A total of 216 samples were included in the analyses; of these, 91 samples representing all 16 sites were stoneroller (only one sample was obtained from Site 7, however), 105 samples from 16 sites were sunfish, and 20 samples representing Sites 4, 7, $8,11,12,13$, and 16 were hog sucker. Censored values $(<\mathrm{LOD})$ were replaced with $50 \%$ of the LOD for statistical computations and graphing. All data representing elemental concentrations were $\log _{10}$-transformed prior to statistical analysis. Percent moisture was transformed using the angular transformation. A preliminary analysis-of-covariance (ANCOVA) was conducted to determine the influence of species, gender, collection site, and the interactions of these variables on metals concentrations and biomarkers. For these analyses, species, gender, and site were considered fixed effects; and total length and fish weight were analyzed as continuous variables. The ANCOVA indicated that metals 
concentrations differed among species and sites. Some interactions of species with other factors were also significant, but few of the other factors included in the models were themselves significant (data not shown). Further statistical analyses were therefore conducted separately for each species. Within species, one-way analysis-of-variance (ANOVA) was used to test for differences among sites and groups of sites, with site considered a fixed effect; differences among individual sites were evaluated with Fisher's protected LSD test. For some analyses, sites were also grouped according to their distance from mining (Table 1): "reference" (no upstream mining - Sites 3, 10, 11, and 15); "near mine" $(<10 \mathrm{~km}$ downstream from a NLB facility - Sites 2, 4, 6, 7, 12, and 14); and "downstream" ( $>10 \mathrm{~km}$ downstream-Sites 1, 5, 8, 9, and 13). Differences among groups of sites were tested as planned non-orthogonal contrasts using single degree-of-freedom $F$-tests. Relations between and among groups of variables (transformed as described previously) were examined with Pearson correlation coefficients and simple linear (least-squares) regression. A significance level of $P<0.05$ was used to judge all statistical tests unless otherwise indicated.

\section{Results}

\subsection{Moisture}

Blood moisture content was similar in all three species, averaging (arithmetic mean, minimum-maximum) 87.4\% $(82.9-93.4 \%)$ in hog sucker, $87.4 \%(83.6-93.5 \%)$ in sunfish, and $86.9 \%(82.7-90.6 \%)$ in stoneroller. Differences among species were not statistically significant $\left(F_{2,135}=0.35, P>0.05\right.$; data not shown $)$, but differences among sites were significant in all species; some site means differed by $>5 \%$ (Table 2). Moisture content in hog sucker and stoneroller from Site 16 (BR) and several sites $<10 \mathrm{~km}$ downstream from NLB mines was significantly greater than from reference and downstream sites, either as individual sites (Table 2) or as groups of sites $(0.1<P<0.05$; data not shown). In sunfish there was no mining-related pattern, and differences among groups of sites based on proximity to mining were not statistically significant (Table 2).

\subsection{Lead}

Lead was detected in 206 of 216 blood samples (95\%) at concentrations of $0.19-7.72 \mu \mathrm{g} / \mathrm{g} \mathrm{dw}$ in hog sucker, $<0.02-$ $3.30 \mu \mathrm{g} / \mathrm{g} \mathrm{dw}$ in stoneroller, and $<0.03-3.80 \mu \mathrm{g} / \mathrm{g} \mathrm{dw}$ in sunfish; all 10 censored values represented sunfish and stoneroller from downstream and reference sites (Fig. 2). Maxima for hog sucker and sunfish were from Site 16 (BR), but maximum concentrations in stoneroller were from Site 14 (Courtois Creek). Concentrations in hog sucker generally exceeded those in sunfish and stoneroller (Table 2). However, and although differences among species were not statistically significant overall $\left(F_{2,}, 135\right.$ $=2.36, P>0.05)$, significant $(P<0.01)$ species $\times$ site interaction indicated that differences were significant at some sites (data not shown). Blood $\mathrm{Pb}$ concentrations in fish of all species from sites $<10 \mathrm{~km}$ downstream from mines and the Big River were significantly $(P<0.01)$ and substantially greater than those from reference and downstream sites (Fig. 2; Table 2). Relative to other sites, blood $\mathrm{Pb}$ concentrations in stoneroller from Site 6 (Logan Creek) and Site 14 were greater than those in sunfish (Table 2); however, the sunfish were collected $2-5 \mathrm{~km}$ downstream of the stoneroller. Concentrations in fish from the exploration area (Site 15) were similar to (sunfish) or less than (stoneroller) those from reference and downstream sites (Table 2). Blood $\mathrm{Pb}$ concentrations in hog sucker and sunfish from downstream and reference sites were not significantly different $(P>0.05)$. All differences among groups of sites were significant $(P<0.01)$ in stoneroller; highest concentrations were in fish from the Big River and sites $<10 \mathrm{~km}$ downstream from mining (Fig. 2).

Lead was detected in 215 of 216 liver samples $(99.5 \%)$ at concentrations of $0.09-17.0 \mu \mathrm{g} / \mathrm{g} \mathrm{dw}$ in hog sucker, $0.005-$ $7.79 \mu \mathrm{g} / \mathrm{g} \mathrm{dw}$ in stoneroller, and $<0.02-3.41 \mu \mathrm{g} / \mathrm{gdw}$ in sunfish (Fig. 2). The hog sucker maximum was a fish from Site 4 (Bee Fork); its concentration was nearly 3-fold higher than that of the next highest in this species $(5.52 \mu \mathrm{g} / \mathrm{g} \mathrm{dw}$, from the Big River). The sunfish maximum was at Site 16 (BR) whereas in stoneroller it was at Site 14 (Courtois Creek). Overall differences among species were statistically significant $\left(F_{2}, \quad 135=3.45, \quad P<0.05\right.$; data not shown $)$; concentrations were generally higher in hog sucker than in sunfish or stoneroller (Table 3). Differences among sites within species were also significant $(P<0.01)$; liver $\mathrm{Pb}$ concentrations in fish of all three species from Site 16 (Big River) and all sites $<10 \mathrm{~km}$ downstream from mines were significantly and substantially greater than those from sites further downstream and reference sites (Fig. 2; Table 3). Liver $\mathrm{Pb}$ concentrations in sunfish from Sites 6 and 14, which were collected several kilometers downstream of the stoneroller, were lower than concentrations in stoneroller relative to other sites (Table 3). As a group, liver $\mathrm{Pb}$ concentrations in hog sucker from sites $<10 \mathrm{~km}$ downstream from NLB mines were not significantly different from the Big River $(P>0.05)$, but all other differences between groups of sites based on proximity to mining were significant $(<0.01$ $<P<0.04$ ). Liver $\mathrm{Pb}$ concentrations in sunfish and stoneroller from sites nearest to mines were also significantly higher $(<0.01<P<0.02)$ than at reference and downstream sites (Table 3).

Concentrations of $\mathrm{Pb}$ in liver were similar to those in blood in all three species and were highly correlated. Statistically significant $(P<0.01) \log -\log$ linear relations with small intercepts and regression coefficients near 1.0 explained $71 \%$ of the variation between blood and liver $\mathrm{Pb}$ in hog sucker, $76 \%$ in stoneroller, and $73 \%$ in sunfish (Fig. 2).

\subsection{Cadmium}

Cadmium was detected in 174 of 216 blood samples $(81 \%)$ at concentrations of $0.008-1.27 \mu \mathrm{g} / \mathrm{g} \mathrm{dw}$ in $\mathrm{hog}$ sucker, $<0.004-0.090 \mu \mathrm{g} / \mathrm{g} \mathrm{dw}$ in stoneroller, and $<0.003-$ $2.44 \mu \mathrm{g} / \mathrm{g} \mathrm{dw}$ in sunfish (Fig. 3). All censored values represented sunfish and stoneroller from reference and 
Table 2

Moisture content (\%) and concentrations of lead, cadmium, and zinc (all $\mu \mathrm{g} / \mathrm{g}$ dry weight) in the blood of three species of fish

\begin{tabular}{|c|c|c|c|c|c|}
\hline Species, site, and (type ${ }^{\mathrm{a}}$ ) & $n$ or df & Moisture & Lead & Cadmium & Zinc \\
\hline \multicolumn{6}{|l|}{ Hog sucker ${ }^{\mathrm{b}}$} \\
\hline $4(\mathrm{M})$ & 2 & $85.1 \pm 0.1 \mathrm{~b}$ & $2.14 \pm 0.82 \mathrm{~b}$ & $0.033 \pm 0.011 \mathrm{bc}$ & $42.6 \pm 5.7 \mathrm{a}$ \\
\hline $7(\mathrm{M})$ & 2 & $90.3 \pm 1.0 \mathrm{a}$ & $3.20 \pm 1.90 \mathrm{~b}$ & $0.745 \pm 0.525 \mathrm{a}$ & $61.2 \pm 6.5 \mathrm{a}$ \\
\hline $12(\mathrm{M})$ & 3 & $87.5 \pm<0.1 \mathrm{ab}$ & $1.37 \pm 0.10 \mathrm{~b}$ & $0.014 \pm 0.001 \mathrm{c}$ & $44.5 \pm 3.3 \mathrm{a}$ \\
\hline 8 (D) & 4 & $85.7 \pm 1.2 \mathrm{~b}$ & $0.20 \pm<0.01 \mathrm{c}$ & $0.068 \pm 0.012 b$ & $48.0 \pm 4.0 \mathrm{a}$ \\
\hline 13 (D) & 3 & $86.7 \pm 0.1 \mathrm{~b}$ & $0.36 \pm 0.03 \mathrm{c}$ & $0.015 \pm 0.002 \mathrm{c}$ & $44.0 \pm 1.9 \mathrm{a}$ \\
\hline $11(\mathrm{R})$ & 3 & $86.0 \pm 0.6 \mathrm{~b}$ & $0.28 \pm 0.04 \mathrm{c}$ & $0.016 \pm 0.007 \mathrm{c}$ & $50.4 \pm 0.5 \mathrm{a}$ \\
\hline $16(\mathrm{BR})$ & 3 & $90.4 \pm 1.7 \mathrm{a}$ & $6.63 \pm 0.87 \mathrm{a}$ & $0.075 \pm 0.028 \mathrm{~b}$ & $48.4 \pm 5.0 \mathrm{a}$ \\
\hline ANOVA- $F$ & $7,19^{\mathrm{c}}$ & $3.54 *$ & $44.00 * *$ & $13.02 * *$ & $1.7 \overline{\mathrm{ns}}$ \\
\hline$R^{2}$ & 20 & 0.62 & 0.95 & 0.86 & 0.45 \\
\hline \multicolumn{6}{|l|}{ Stoneroller $^{\mathrm{d}}$} \\
\hline $2(\mathrm{M})$ & 7 & $87.0 \pm 0.5 \mathrm{~b}$ & $0.33 \pm 0.02 \mathrm{efg}$ & $0.023 \pm 0.009 \mathrm{cdef}$ & $51.2 \pm 2.6 \mathrm{de}$ \\
\hline $4(\mathrm{M})$ & 6 & $84.6 \pm 0.3 \mathrm{c}$ & $0.98 \pm 0.08 \mathrm{bc}$ & $0.015 \pm 0.003 \mathrm{defg}$ & $40.8 \pm 2.4 \mathrm{f}$ \\
\hline $6(\mathrm{M})$ & 3 & $87.9 \pm 0.5 \mathrm{ab}$ & $0.75 \pm 0.11 \mathrm{bcd}$ & $0.043 \pm 0.007 \mathrm{a}$ & $58.9 \pm 5.3$ bcde \\
\hline $12(\mathrm{M})$ & 6 & $87.1 \pm 0.4 \mathrm{ab}$ & $0.62 \pm 0.05 \mathrm{~cd}$ & $0.010 \pm 0.002 \mathrm{fg}$ & $64.5 \pm 4.2 \mathrm{bc}$ \\
\hline $14(\mathrm{M})$ & 6 & $87.1 \pm 0.5 \mathrm{ab}$ & $2.25 \pm 0.33 \mathrm{a}$ & $0.029 \pm 0.004 \mathrm{abc}$ & $58.4 \pm 5.0$ cde \\
\hline $1(\mathrm{D})$ & 7 & $88.5 \pm 0.4 \mathrm{a}$ & $0.19 \pm 0.01 \mathrm{gh}$ & $0.032 \pm 0.007 \mathrm{abcd}$ & $69.3 \pm 1.6 \mathrm{ab}$ \\
\hline 5 (D) & 6 & $87.9 \pm 0.4 \mathrm{ab}$ & $0.12 \pm 0.02 \mathrm{ij}$ & $0.014 \pm 0.006 \mathrm{efg}$ & $64.5 \pm 2.5 \mathrm{bc}$ \\
\hline 8 (D) & 6 & $86.5 \pm 0.8 \mathrm{~b}$ & $0.15 \pm 0.02 \mathrm{hi}$ & $0.038 \pm 0.012 \mathrm{ab}$ & $64.0 \pm 3.6 \mathrm{bc}$ \\
\hline 9 (D) & 6 & $87.7 \pm 0.8 \mathrm{ab}$ & $0.30 \pm 0.05 \mathrm{fg}$ & $0.020 \pm 0.007$ bcdef & $69.5 \pm 2.9 \mathrm{ab}$ \\
\hline 13 (D) & 6 & $88.5 \pm 0.3 \mathrm{a}$ & $0.52 \pm 0.06 \mathrm{de}$ & $0.008 \pm \pm 0.001 \mathrm{fg}$ & $57.8 \pm 2.4 \mathrm{cde}$ \\
\hline $3(\mathrm{R})$ & 6 & $87.7 \pm 0.3 \mathrm{ab}$ & $0.12 \pm 0.04 \mathrm{ij}$ & $0.032 \pm 0.009$ abcde & $59.7 \pm 5.2 \mathrm{bcd}$ \\
\hline $10(\mathrm{R})$ & 7 & $87.3 \pm 0.3 \mathrm{ab}$ & $0.03 \pm 0.01 \mathrm{k}$ & $0.010 \pm 0.001 \mathrm{fg}$ & $49.3 \pm 2.1 \mathrm{e}$ \\
\hline $11(\mathrm{R})$ & 6 & $87.6 \pm 0.5 \mathrm{ab}$ & $0.35 \pm 0.02$ ef & $0.008 \pm 0.002 \mathrm{~g}$ & $63.7 \pm 5.2 \mathrm{bc}$ \\
\hline 15 (R/EA) & 6 & $84.4 \pm 0.8 \mathrm{c}$ & $0.12 \pm 0.05 \mathrm{j}$ & $0.007 \pm 0.002 \mathrm{~g}$ & $51.0 \pm 3.0 \mathrm{de}$ \\
\hline $16(\mathrm{BR})$ & 7 & $83.7 \pm 0.5 \mathrm{c}$ & $1.39 \pm 0.25 \mathrm{ab}$ & $0.022 \pm 0.003$ abcde & $82.5 \pm 5.5 \mathrm{a}$ \\
\hline ANOVA- $F$ & $14,90^{\mathrm{c}}$ & $8.33 * *$ & $37.43 * *$ & $4.67 * *$ & $7.97 * *$ \\
\hline$R^{2}$ & 91 & 0.61 & 0.87 & 0.46 & 0.59 \\
\hline \multicolumn{6}{|l|}{ Sunfish ${ }^{\mathrm{e}}$} \\
\hline $2(\mathrm{M})$ & 10 & $87.8 \pm 0.3 \mathrm{abc}$ & $0.44 \pm 0.05 \mathrm{c}$ & $0.018 \pm 0.009 \mathrm{cdef}$ & $47.7 \pm 2.4 \mathrm{~cd}$ \\
\hline $4(\mathrm{M})$ & 8 & $87.2 \pm 0.5 \mathrm{bcd}$ & $1.13 \pm 0.90 \mathrm{~b}$ & $0.021 \pm 0.004 \mathrm{abcd}$ & $51.4 \pm 1.7 \mathrm{~cd}$ \\
\hline $6(\mathrm{M})$ & 6 & $88.0 \pm 0.5 \mathrm{abc}$ & $0.32 \pm 0.04 \mathrm{de}$ & $0.042 \pm 0.018 \mathrm{abc}$ & $43.2 \pm 2.2 \mathrm{~d}$ \\
\hline $7(\mathrm{M})$ & 6 & $88.3 \pm 1.1 \mathrm{ab}$ & $1.32 \pm 0.30 \mathrm{~b}$ & $0.026 \pm 0.004 \mathrm{abc}$ & $53.9 \pm 2.8 \mathrm{~cd}$ \\
\hline $12(\mathrm{M})$ & 6 & $88.4 \pm 0.4 \mathrm{ab}$ & $0.62 \pm 0.14 \mathrm{c}$ & $0.005 \pm 0.001$ efg & $69.4 \pm 3.6 \mathrm{ab}$ \\
\hline $14(\mathrm{M})$ & 6 & $86.7 \pm 0.4$ cde & $0.64 \pm 0.19 \mathrm{cf}$ & $0.006 \pm 0.001 \mathrm{defg}$ & $47.4 \pm 2.5 \mathrm{~cd}$ \\
\hline 1 (D) & $7^{\mathrm{f}}$ & $87.8 \pm 0.4 \mathrm{abc}$ & $0.16 \pm 0.02 \mathrm{f}$ & $0.016 \pm 0.005$ bcde & $62.8 \pm 4.1 \mathrm{a}$ \\
\hline 5 (D) & $6^{\mathrm{g}}$ & $88.4 \pm 0.6 \mathrm{ab}$ & $0.39 \pm 0.10 \mathrm{cde}$ & $0.021 \pm 0.007 \mathrm{a}$ & $43.3 \pm 2.6 \mathrm{~d}$ \\
\hline 8 (D) & 6 & $85.2 \pm 0.5 \mathrm{e}$ & $0.09 \pm 0.10 \mathrm{~g}$ & $0.033 \pm 0.016 \mathrm{abcd}$ & $50.9 \pm 1.9 \mathrm{~cd}$ \\
\hline 9 (D) & 6 & $87.4 \pm 0.6 \mathrm{abc}$ & $0.17 \pm 0.03 \mathrm{f}$ & $0.007 \pm 0.004$ efg & $49.9 \pm 3.5 \mathrm{~cd}$ \\
\hline 13 (D) & 6 & $86.9 \pm 0.4 \mathrm{~cd}$ & $0.18 \pm 0.04 \mathrm{f}$ & $0.002 \pm<0.001 \mathrm{~g}$ & $45.4 \pm 1.1 \mathrm{~d}$ \\
\hline $3(\mathrm{R})$ & 6 & $88.8 \pm 0.6 \mathrm{a}$ & $0.09 \pm 0.02 \mathrm{~g}$ & $0.021 \pm 0.003 \mathrm{abc}$ & $59.6 \pm 3.8 \mathrm{bc}$ \\
\hline $10(\mathrm{R})$ & 7 & $85.7 \pm 0.4 \mathrm{de}$ & $0.09 \pm 0.03 \mathrm{~g}$ & $0.030 \pm 0.023 \mathrm{cde}$ & $50.7 \pm 2.2 \mathrm{~cd}$ \\
\hline $11(\mathrm{R})$ & 6 & $88.1 \pm 0.3 \mathrm{abc}$ & $0.38 \pm 0.07$ cde & $0.003 \pm 0.001 \mathrm{fg}$ & $44.5 \pm 3.3 \mathrm{~d}$ \\
\hline 15 (R/EA) & 6 & $86.6 \pm 0.4$ cde & $0.24 \pm 0.04$ ef & $0.004 \pm 0.002 \mathrm{fg}$ & $52.7 \pm 2.5 \mathrm{~cd}$ \\
\hline $16(\mathrm{BR})$ & 7 & $87.1 \pm 0.6 \mathrm{bcd}$ & $2.65 \pm 0.33 \mathrm{a}$ & $0.029 \pm 0.004 \mathrm{ab}$ & $51.1 \pm 3.8 \mathrm{~cd}$ \\
\hline ANOVA- $F$ & $15,89^{\mathrm{c}}$ & $3.52 * *$ & $29.56 * *$ & $5.43 * *$ & $3.84 * *$ \\
\hline$R^{2}$ & $105^{\mathrm{h}}$ & 0.37 & 0.83 & 0.48 & 0.39 \\
\hline
\end{tabular}

Shown are arithmetic site means $\pm \mathrm{SE}$, numbers of observations $(n)$, and results of one-way analysis-of-variance (ANOVA) as $F$-values $(* * P<0.01$, $* P<0.05$, ns $P>0.05$ ), degrees-of-freedom (df), and coefficients of determination $\left(R^{2}\right)$. Within species, means followed by the same letter (ranked alphabetically from highest to lowest) are not significantly different (Fisher's protected LSD, $P>0.05$ ).

${ }^{\mathrm{a}} \mathrm{M},<10 \mathrm{~km}$ downstream of New Lead Belt mine; D, $>10 \mathrm{~km}$ downstream; R, upstream of all mining (reference); EA, exploration area; BR, Big River.

${ }^{\mathrm{b}}$ Northern hog sucker, Hypentelium nigricans.

${ }^{\mathrm{c}} \mathrm{df}$.

${ }^{\mathrm{d}}$ Largescale stoneroller, Campostoma oligolepis.

${ }^{\mathrm{e}}$ Longear sunfish, Lepomis megalotis.

${ }^{\mathrm{f}} 6$ for zinc.

$\mathrm{g}_{5}$ for cadmium.

${ }^{\mathrm{h}} 104$ for cadmium and zinc.

downstream sites (Fig. 3). The sample representing the sunfish maximum $(2.44 \mu \mathrm{g} / \mathrm{gdw}$, from Site 5) was reanalyzed and found to contain $2.62 \mu \mathrm{g} / \mathrm{g} \mathrm{dw}$. Because this is more than 5 -fold greater than typical blood $\mathrm{Cd}$ concentrations in fish from $\mathrm{Pb}-\mathrm{Zn}$ mining areas (e.g., Brumbaugh et al., 2005), external contamination was suspected and this sample was excluded from all subsequent computations involving blood $\mathrm{Cd}$. Without this 

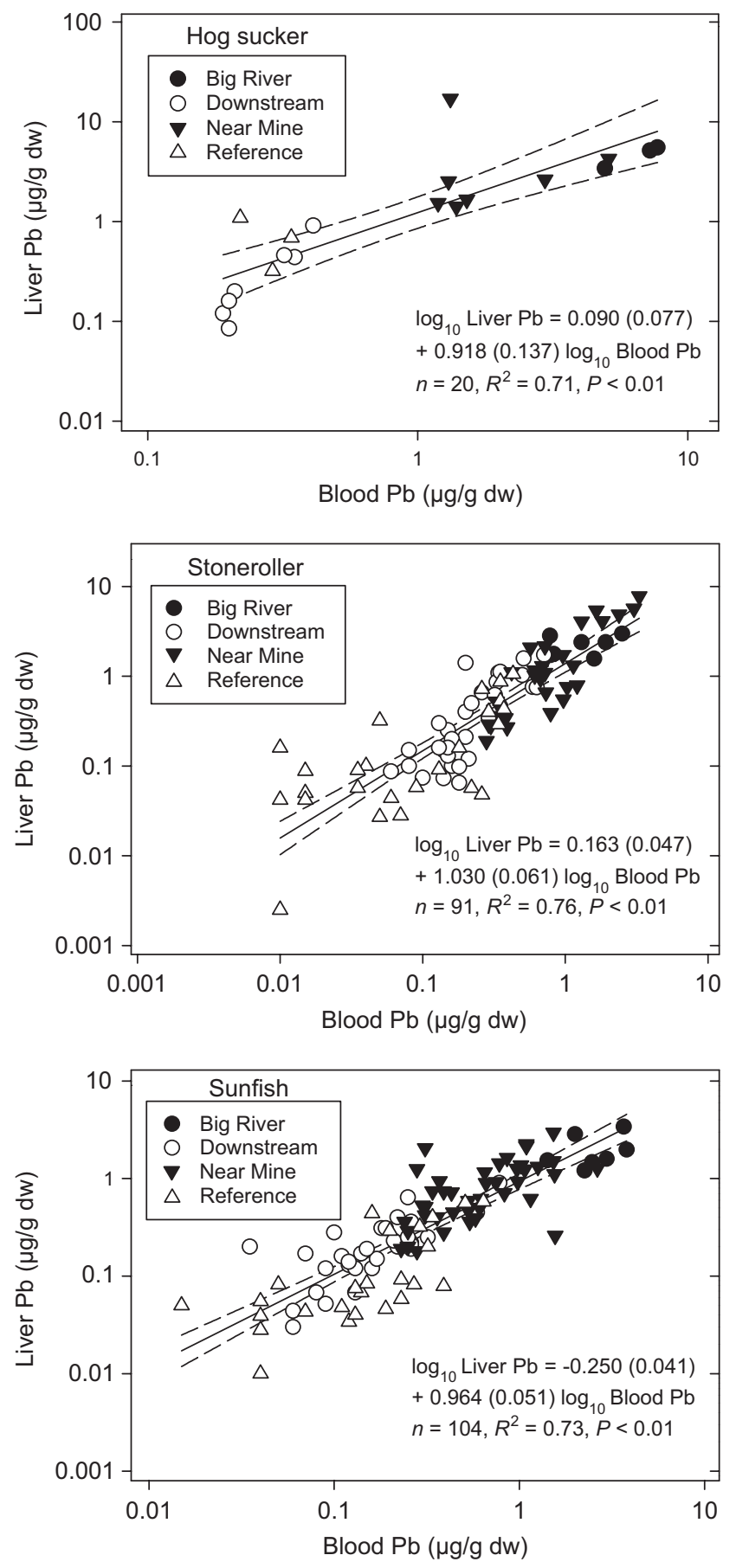

Fig. 2. Blood and liver concentrations of lead $(\mathrm{Pb})$ in three species of fish from sites $<10 \mathrm{~km}$ downstream from mining-related facilities in the New Lead Belt (Near Mines), $>10 \mathrm{~km}$ downstream (Downstream), no upstream mining (Reference), and the Big River. Also shown for each species are the least-squares regressions (solid lines) and $95 \%$ confidence intervals (dashed lines) between these variables across all sites (standard errors in parentheses).

extreme value the greatest blood $\mathrm{Cd}$ concentration in sunfish was $0.110 \mu \mathrm{g} / \mathrm{gdw}$ (at Site 1, West Fork at Sutton Bluff). Blood $\mathrm{Cd}$ concentrations were significantly $(P<0.01)$ greater in hog sucker (data not shown). Differences among sites were statistically significant
$(P<0.01)$ in all three species (Table 2), but blood $\mathrm{Cd}$ differences were less apparent than those for blood $\mathrm{Pb}$. Concentrations in hog sucker from several sites near NLB mines and the Big River (Sites 7, 12 and 16) were significantly greater than those from downstream and reference sites (Sites 11 and 13), but concentrations in hog sucker from other sites near mines and downstream were intermediate (Table 2). Nevertheless, all differences among groups of sites were significant $(<0.01<P<0.05)$ except for those between sites $<10 \mathrm{~km}$ downstream of NLB mines and the Big River $(P>0.05)$. Trends were less evident in sunfish and stoneroller; although blood $\mathrm{Cd}$ concentrations in fish from the Big River (Site 16) were elevated relative to most other sites, concentrations were also comparatively high at some downstream and reference sites and low at some sites near mines (Fig. 3; Table 2). Concentrations were also relatively high in sunfish from Sites 6 and 14 even though they were collected $2-5 \mathrm{~km}$ downstream of the stoneroller (Table 2). Among groups of sites, blood Cd in sunfish from sites $<10 \mathrm{~km}$ downstream from mines, $>10 \mathrm{~km}$ downstream, and the Big River were not significantly different $(P>0.05)$; however, and in spite of the variation among individual site means, concentrations in sunfish representing all three mining-affected groups of sites were significantly greater than at reference sites $(<0.01<P<0.03)$. Concentrations in stoneroller and sunfish from Site 15 (exploration area) were similar to those from reference and downstream sites (Table 2).

Cadmium concentrations in liver were greater than those in blood; consequently, $\mathrm{Cd}$ was detected in all 216 liver samples at concentrations of $0.310-20.7 \mu \mathrm{g} / \mathrm{g} \mathrm{dw}$ in hog sucker, $0.480-30.6 \mu \mathrm{g} / \mathrm{g} \mathrm{dw}$ in sunfish, and $0.060-11.7 \mu \mathrm{g} /$ $\mathrm{gdw}$ in stoneroller (Fig. 3). The hog sucker maximum was the same fish from Site 4 that represented the liver $\mathrm{Pb}$ maximum; the next greatest value $(13.0 \mu \mathrm{g} / \mathrm{g} \mathrm{dw})$ was from Site 7 (Sweetwater Creek). The stoneroller and sunfish maxima were fish from Site 14, the latter despite the fact that the sunfish were collected $2-5 \mathrm{~km}$ downstream of the stonerollers. Overall differences among species were not statistically significant $\left(F_{2}, 135=1.27, P>0.05\right)$; leastsquares mean concentrations were $4.59 \mu \mathrm{g} / \mathrm{gdw}$ in hog sucker, $4.27 \mu \mathrm{g} / \mathrm{g} \mathrm{dw}$ in sunfish, and $1.03 \mu \mathrm{g} / \mathrm{g} \mathrm{dw}$ in stoneroller. However, significant $(P<0.01)$ species $\times$ site interaction in all species indicated differences at some sites. Consequently, liver $\mathrm{Cd}$ differences among sites were highly significant $(P<0.01)$ in all three species (Table 3$)$. Concentrations in hog sucker were highest at Sites 4 and 7 , in the NLB, and were also lowest at Site 12, which is also in the NLB (Fig. 3; Table 3). All other sites, including the $\mathrm{BR}$, were intermediate. Consequently, liver Cd concentrations in hog sucker from sites $<10 \mathrm{~km}$ downstream of NLB mines were significantly $(P<0.05)$ greater than those from the Big River, but no other differences between groups of sites were significant $(P>0.05)$. In contrast, all differences between groups of sites were highly significant $(P<0.01)$ in sunfish except for those between reference and downstream sites $(P>0.05)$; concentrations in fish from sites near mines 
Table 3

Concentrations of lead, cadmium, and zinc (all $\mu \mathrm{g} / \mathrm{g}$ dry weight) in the livers of three species of fish

\begin{tabular}{|c|c|c|c|c|}
\hline Species, site, and (type) ${ }^{\mathrm{a}}$ & $n$ or df & Lead & Cadmium & Zinc \\
\hline \multicolumn{5}{|l|}{ Hog sucker ${ }^{\mathrm{b}}$} \\
\hline $4(\mathrm{M})$ & 2 & $9.82 \pm 7.19 \mathrm{a}$ & $11.1 \pm 9.60 \mathrm{ab}$ & $733 \pm 567 a$ \\
\hline $7(\mathrm{M})$ & 2 & $3.39 \pm 0.86 \mathrm{ab}$ & $10.6 \pm 2.38 \mathrm{a}$ & $168 \pm 8.0 \mathrm{~b}$ \\
\hline $12(\mathrm{M})$ & 3 & $1.53 \pm 0.07 \mathrm{bc}$ & $0.52 \pm 0.12 \mathrm{~d}$ & $138 \pm 14.2 \mathrm{bc}$ \\
\hline 8 (D) & 4 & $0.14 \pm 0.02 \mathrm{e}$ & $4.30 \pm 1.08 \mathrm{abc}$ & $149 \pm 3.4 \mathrm{bc}$ \\
\hline 13 (D) & 3 & $0.60 \pm 0.15 \mathrm{~d}$ & $1.41 \pm 0.17 \mathrm{~cd}$ & $135 \pm 14.6 \mathrm{bc}$ \\
\hline $11(\mathrm{R})$ & 3 & $0.70 \pm 0.22 \mathrm{~cd}$ & $1.77 \pm 0.87 \mathrm{~cd}$ & $116 \pm 5.5 \mathrm{bc}$ \\
\hline $16(\mathrm{BR})$ & 3 & $4.70 \pm 0.65 \mathrm{a}$ & $2.34 \pm 0.09 \mathrm{bc}$ & $74.8 \pm 10.5 \mathrm{c}$ \\
\hline ANOVA- $F$ & $6,13^{\mathrm{e}}$ & $21.61 * *$ & $5.26 * *$ & $3.93 *$ \\
\hline$R^{2}$ & 20 & 0.91 & 0.89 & 0.64 \\
\hline \multicolumn{5}{|l|}{ Stoneroller ${ }^{\mathrm{c}}$} \\
\hline $2(\mathrm{M})$ & 7 & $0.32 \pm 0.04 \mathrm{ef}$ & $0.25 \pm 0.07$ ef & $89.0 \pm 2.4 \mathrm{bcd}$ \\
\hline $4(\mathrm{M})$ & 6 & $0.75 \pm 0.13 \mathrm{~cd}$ & $0.40 \pm 0.10 \mathrm{bcd}$ & $46.0 \pm 4.9 \mathrm{e}$ \\
\hline $6(\mathrm{M})$ & 3 & $1.23 \pm 0.25 \mathrm{bcd}$ & $4.06 \pm 0.10 \mathrm{a}$ & $103 \pm 4 b c$ \\
\hline $12(\mathrm{M})$ & 6 & $1.49 \pm 0.20 \mathrm{bc}$ & $0.35 \pm 0.05 \mathrm{bcd}$ & $109 \pm 9 \mathrm{abc}$ \\
\hline $14(\mathrm{M})$ & 6 & $5.32 \pm 0.56 \mathrm{a}$ & $3.91 \pm 1.62 \mathrm{a}$ & $130 \pm 11.5 \mathrm{a}$ \\
\hline 1 (D) & 7 & $0.27 \pm 0.08 \mathrm{fg}$ & $1.08 \pm 0.24 \mathrm{~b}$ & $95.6 \pm 6.7 \mathrm{bcd}$ \\
\hline 5 (D) & 6 & $0.12 \pm 0.01 \mathrm{ghi}$ & $0.28 \pm 0.06 \mathrm{de}$ & $93.6 \pm 3.9 \mathrm{bcd}$ \\
\hline 8 (D) & 6 & $0.18 \pm 0.07 \mathrm{gh}$ & $0.33 \pm 0.07 \mathrm{cde}$ & $116.6 \pm 22.0 \mathrm{ab}$ \\
\hline 9 (D) & 6 & $0.84 \pm 0.18 \mathrm{~cd}$ & $0.92 \pm 0.19 \mathrm{~b}$ & $127.0 \pm 3.5 \mathrm{a}$ \\
\hline 13 (D) & 6 & $1.09 \pm 0.19 \mathrm{~cd}$ & $0.58 \pm 0.05 \mathrm{bc}$ & $101.8 \pm 5.5 \mathrm{bc}$ \\
\hline $3(\mathrm{R})$ & 6 & $0.11 \pm 0.06 \mathrm{ij}$ & $1.03 \pm 0.28 \mathrm{~b}$ & $88.2 \pm 3.2 \mathrm{~cd}$ \\
\hline $10(\mathrm{R})$ & 7 & $0.11 \pm 0.04 \mathrm{hij}$ & $1.02 \pm 0.20 \mathrm{~b}$ & $89.8 \pm 2.1 \mathrm{bcd}$ \\
\hline $11(\mathrm{R})$ & 6 & $0.65 \pm 0.12 \mathrm{de}$ & $0.60 \pm 0.15 b c$ & $101 \pm 8.0 \mathrm{bc}$ \\
\hline $15(\mathrm{R} / \mathrm{EA})$ & 6 & $0.08 \pm 0.03 \mathrm{j}$ & $0.20 \pm 0.13 \mathrm{f}$ & $89.9 \pm 3.4$ bcd \\
\hline $16(\mathrm{BR})$ & 7 & $2.23 \pm 0.21 b$ & $0.51 \pm 0.07 \mathrm{bcd}$ & $80.8 \pm 2.7 \mathrm{~d}$ \\
\hline ANOVA- $F$ & $14,76^{\mathrm{e}}$ & $30.93 * *$ & $12.77 * *$ & $11.49 * *$ \\
\hline$R^{2}$ & 91 & 0.85 & 0.70 & 0.68 \\
\hline \multicolumn{5}{|l|}{ Sunfish ${ }^{\mathrm{d}}$} \\
\hline $2(\mathrm{M})$ & 10 & $0.46 \pm 0.04 \mathrm{ef}$ & $1.97 \pm 0.35 \mathrm{gh}$ & $95.7 \pm 3.2 \mathrm{abc}$ \\
\hline $4(\mathrm{M})$ & 8 & $1.76 \pm 0.23 \mathrm{ab}$ & $4.20 \pm 1.07 \mathrm{def}$ & $87.5 \pm 2.6 \mathrm{bc}$ \\
\hline $6(\mathrm{M})$ & 6 & $0.44 \pm 0.10$ ef & $5.10 \pm 0.40 \mathrm{bcd}$ & $102 \pm 3.7 \mathrm{abc}$ \\
\hline $7(\mathrm{M})$ & 6 & $1.03 \pm 0.16 \mathrm{bc}$ & $9.22 \pm 2.66 \mathrm{ab}$ & $113 \pm 23.5 \mathrm{abc}$ \\
\hline $12(\mathrm{M})$ & 6 & $1.17 \pm 0.22 \mathrm{bc}$ & $5.84 \pm 1.92 \mathrm{cde}$ & $97.3 \pm 6.1 \mathrm{abc}$ \\
\hline $14(\mathrm{M})$ & 6 & $0.62 \pm 0.14 \mathrm{de}$ & $8.49 \pm 4.45 \mathrm{abcd}$ & $89.5 \pm 9.4$ bc \\
\hline 1 (D) & 7 & $0.14 \pm 0.01 \mathrm{hi}$ & $1.31 \pm 0.28 \mathrm{ij}$ & $110 \pm 11.2 \mathrm{ab}$ \\
\hline 5 (D) & 6 & $0.44 \pm 0.12$ ef & $2.56 \pm 0.31 \mathrm{efg}$ & $87.4 \pm 3.7 \mathrm{bc}$ \\
\hline 8 (D) & 6 & $0.08 \pm 0.02 \mathrm{jk}$ & $2.30 \pm 0.49 \mathrm{efgh}$ & $81.9 \pm 8.1 \mathrm{~cd}$ \\
\hline 9 (D) & 6 & $0.24 \pm 0.04 \mathrm{gh}$ & $2.30 \pm 0.56 \mathrm{efg}$ & $119 \pm 8.6 \mathrm{a}$ \\
\hline 13 (D) & 6 & $0.27 \pm 0.02 \mathrm{fg}$ & $2.42 \pm 0.48 \mathrm{efg}$ & $87.2 \pm 4.7 \mathrm{bc}$ \\
\hline $3(\mathrm{R})$ & 6 & $0.03 \pm 0.011$ & $1.77 \pm 0.12 \mathrm{gh}$ & $85.3 \pm 7.5 \mathrm{bcd}$ \\
\hline $10(\mathrm{R})$ & 7 & $0.06 \pm 0.01 \mathrm{k}$ & $1.56 \pm 0.34 \mathrm{ghi}$ & $86.4 \pm 4.6$ bc \\
\hline $11(\mathrm{R})$ & 6 & $0.39 \pm 0.06 \mathrm{efg}$ & $7.54 \pm 1.18 \mathrm{abc}$ & $105 \pm 4.4 \mathrm{abc}$ \\
\hline $15(\mathrm{R} / \mathrm{EA})$ & 6 & $0.14 \pm 0.06 \mathrm{ij}$ & $0.91 \pm 0.19 \mathrm{j}$ & $83.9 \pm 5.4$ bcd \\
\hline $16(\mathrm{BR})$ & 7 & $2.01 \pm 0.31 \mathrm{a}$ & $10.89 \pm 1.43 \mathrm{a}$ & $65.0 \pm 3.9 \mathrm{~d}$ \\
\hline ANOVA- $F$ & $15,89^{\mathrm{e}}$ & $40.61 * *$ & $10.88 * *$ & $2.01 *$ \\
\hline$R^{2}$ & 105 & 0.89 & 0.65 & 0.25 \\
\hline
\end{tabular}

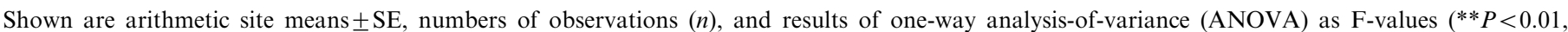

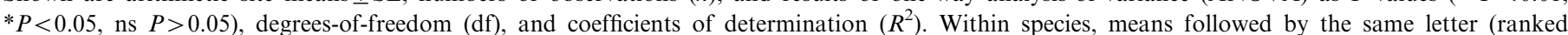
alphabetically from highest to lowest) are not significantly different (Fisher's protected LSD, $P>0.05$ ).

${ }^{a} \mathrm{M},<10 \mathrm{~km}$ downstream of New Lead Belt mine; D, $>10 \mathrm{~km}$ downstream; R, upstream of all mining (reference); EA, exploration area; BR, Big River.

${ }^{\mathrm{b}}$ Northern hog sucker, Hypentelium nigrican.s.

${ }^{\mathrm{c}}$ Largescale stoneroller, Campostoma oligolepis.

${ }^{\mathrm{d}}$ Longear sunfish, Lepomis megalotis.

${ }^{\mathrm{e}} \mathrm{df}$.

were highest. Liver $\mathrm{Cd}$ concentrations in stoneroller from sites $<10 \mathrm{~km}$ from NLB mines were also significantly greater than at downstream sites $(P<0.05)$, but no other differences between groups of sites were significant
$(P>0.05)$. Liver $\mathrm{Cd}$ concentrations in sunfish from Site 6 were high relative to most sites, but those from Site 14 were low; these fish were collected several kilometers downstream of the stonerollers. In sunfish, liver $\mathrm{Cd}$ 

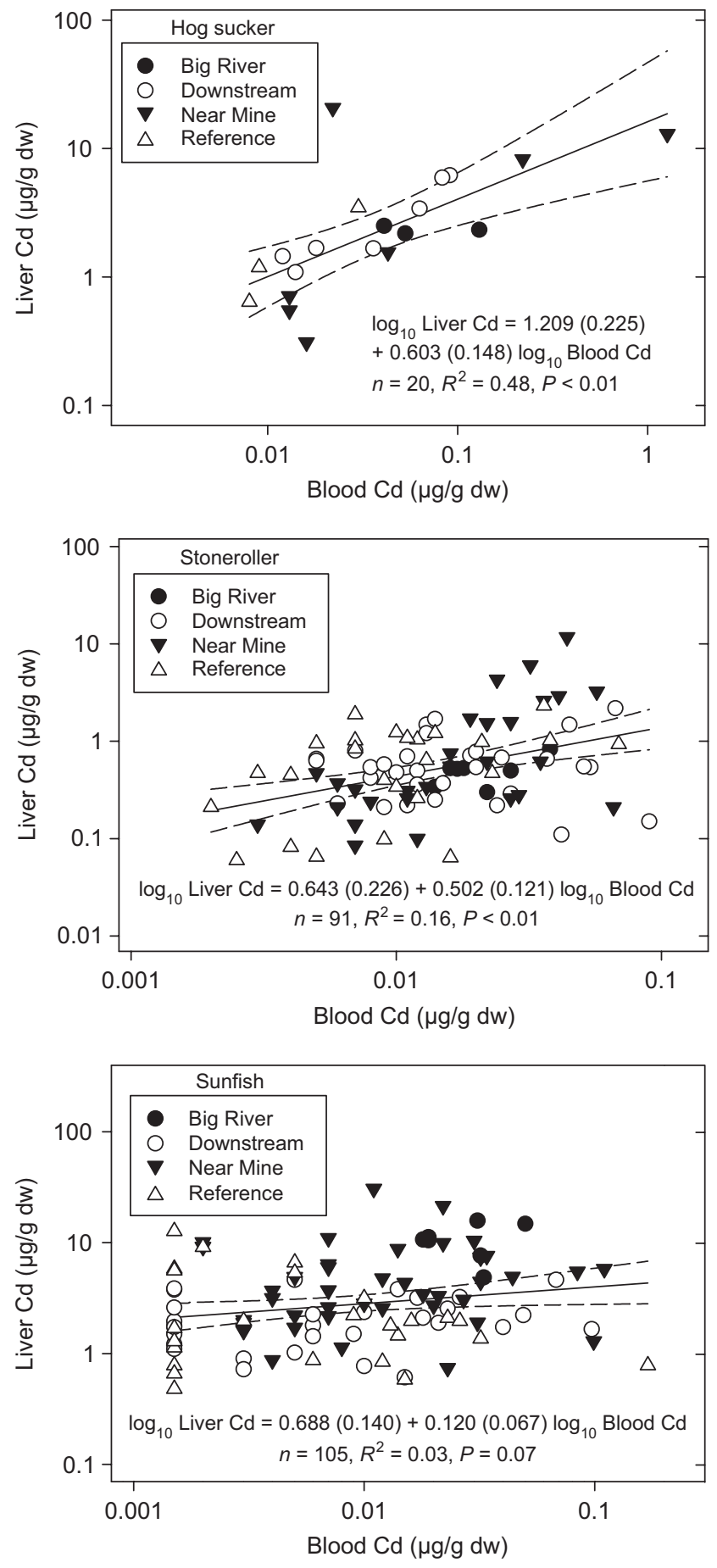

Fig. 3. Blood and liver concentrations of cadmium (Cd) in three species of fish from sites $<10 \mathrm{~km}$ downstream from mining-related facilities in the New Lead Belt (Near Mines), $>10 \mathrm{~km}$ downstream (Downstream), no upstream mining (Reference), and the Big River. Also shown for each species are the least-squares regressions (solid lines) and $95 \%$ confidence intervals (dashed lines) between these variables across all sites (standard errors in parentheses).

concentrations were highest at Site 16 (BR) and at several mining-affected sites in the NLB and lowest at Site 15 (Eleven Point River, in the exploration area) and reference sites. Concentrations in stoneroller were also highest at several NLB sites and lowest at Site 15, with those from the Big River intermediate (Table 3).

Dry-weight blood and liver $\mathrm{Cd}$ concentrations were positively correlated in all three species (Fig. 3), but not as strongly as $\mathrm{Pb}$ (Fig. 2). Log-log linear regressions explained $48 \%$ of the Cd variation in hog sucker, but only $16 \%$ in stoneroller and $3 \%$ in sunfish; the latter only approached statistical significance $(P=0.07)$, partly due to the large number of censored blood $\mathrm{Cd}$ values (Fig. 3). Conversely, the correlation for hog sucker was negatively affected by the one large liver $\mathrm{Cd}$ value representing the fish from Site 4. Regardless, all regression coefficients were positive and the intercepts were large relative to the relationships for $\mathrm{Pb}$, reflecting the substantially greater $\mathrm{dw}$ concentrations of $\mathrm{Cd}$ in liver than in blood (Fig. 3).

\subsection{Zinc}

Zinc was detected in all 216 blood samples analyzed at concentrations of $34.0-99.7 \mu \mathrm{g} / \mathrm{g} \mathrm{dw}$ in stoneroller, $36.9-67.6 \mu \mathrm{g} / \mathrm{g} \mathrm{dw}$ in hog sucker, and $30.8-340 \mu \mathrm{g} / \mathrm{g} \mathrm{dw}$ in sunfish (Fig. 4). The sample representing the extreme value in sunfish $(340 \mu \mathrm{g} / \mathrm{g} \mathrm{dw}$, from Site 1) was re-analyzed and found to contain $401 \mu \mathrm{g} / \mathrm{g} \mathrm{dw}$. Because $300-400 \mu \mathrm{g} / \mathrm{g} \mathrm{dw}$ is about 5 -fold greater than typical blood $\mathrm{Zn}$ concentrations in fish from $\mathrm{Pb}-\mathrm{Zn}$ mining areas (e.g., Brumbaugh et al., 2005), external contamination was suspected and this sample was excluded from all subsequent computations involving blood $\mathrm{Zn}$. The next highest concentration in sunfish was $80.4 \mu \mathrm{g} / \mathrm{g} \mathrm{dw}$ in a sample from Site 12 (Strother Creek; Fig. 4). Blood Zn concentrations were similar in all three species, and differences among species were not statistically significant $\left(F_{2,135}=0.08, P>0.01\right)$. However, as was also true for blood $\mathrm{Pb}$ and $\mathrm{Cd}$, significant $(P<0.01)$ species $\times$ site interaction indicated among-species differences at some sites. Differences among sites were significant in sunfish and stoneroller $(P<0.01)$, but not in hog sucker $(P>0.05)$. Blood $\mathrm{Zn}$ concentrations in stoneroller were significantly greater at Site 16 (BR) than at most other sites and groups of sites. Conversely, blood $\mathrm{Zn}$ concentrations in stoneroller were significantly lower at Site 4 (Bee Fork) than at all other sites, including the reference sites (Table 2). However, no general mining-related patterns were evident in any species (Table 2). Blood $\mathrm{Zn}$ concentrations were comparatively low in sunfish from Sites 6 and 14, which were collected several kilometers downstream of the stonerollers.

Zinc also was detected in all 216 liver samples analyzed. The range of concentrations in liver were much greater than in blood; concentrations were $59.3-1300 \mu \mathrm{g} / \mathrm{gdw}$ in hog sucker, $18.8-195 \mu \mathrm{g} / \mathrm{g} \mathrm{dw}$ in sunfish, and $33.1-219 \mu \mathrm{g} / \mathrm{g} \mathrm{dw}$ in stoneroller (Fig. 4). The hog sucker maximum was the same fish from Site 4 that represented the maxima for $\mathrm{Pb}$ and $\mathrm{Cd}$; the next greatest value $(176 \mu \mathrm{g} / \mathrm{gdw})$ was from Site 7 (Sweetwater Creek), as was true for liver Cd. The liver $\mathrm{Zn}$ maxima for sunfish and stoneroller were also from Site 7; the next lowest value was $52.1 \mu \mathrm{g} / \mathrm{g} \mathrm{dw}$ in a fish from 

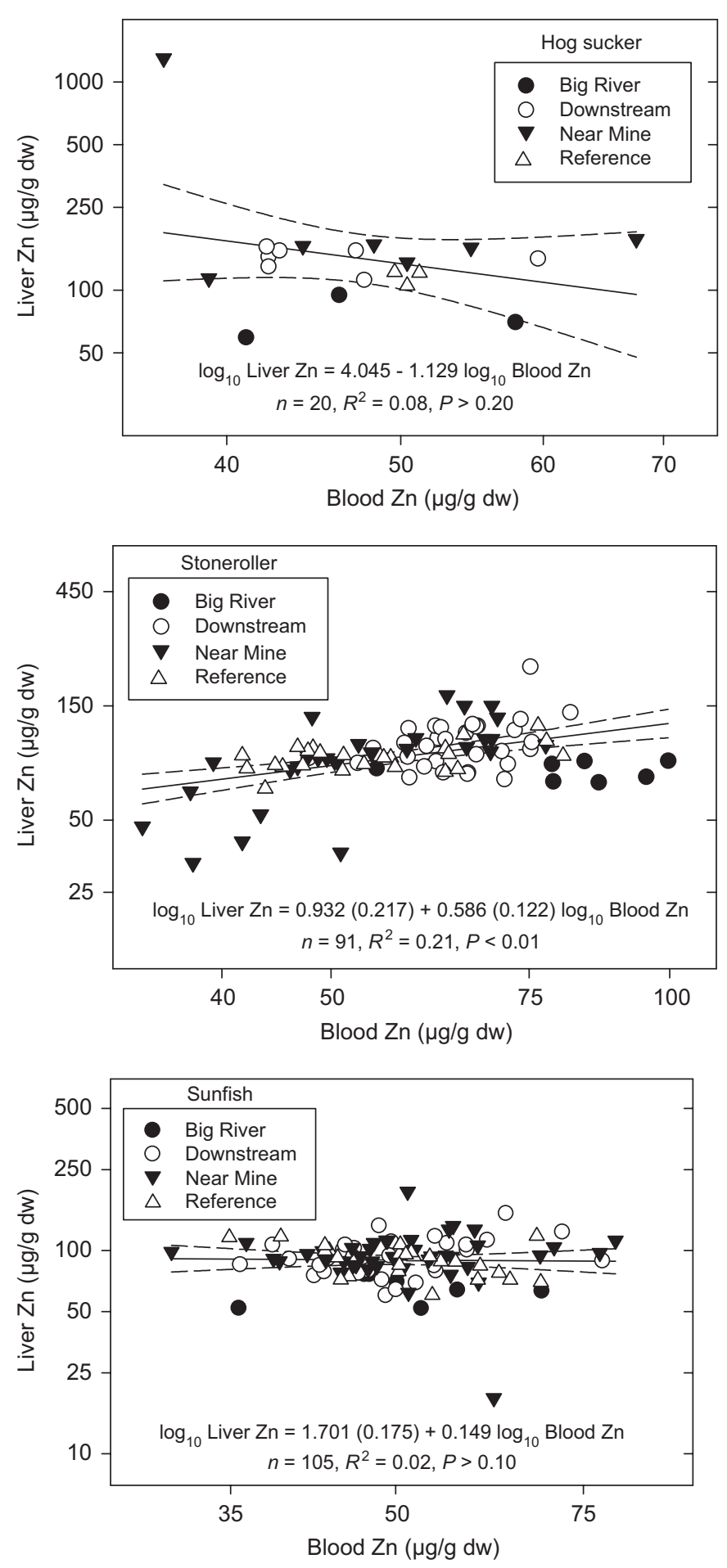

Fig. 4. Blood and liver concentrations of zinc $(\mathrm{Zn})$ in three species of fish from sites $<10 \mathrm{~km}$ downstream from mining-related facilities in the New Lead Belt (Near Mines), $>10 \mathrm{~km}$ downstream (Downstream), no upstream mining (Reference), and the Big River. Also shown for each species are the least-squares regressions (solid lines) and 95\% confidence intervals (dashed lines) between these variables across all sites (standard errors in parentheses).

Site 16 (Big River). However, the sunfish minimum $(18.8 \mu \mathrm{g} / \mathrm{g} \mathrm{dw})$ was also from Site 7. Overall, among-species differences in liver $\mathrm{Zn}$ were not statistically significant
$\left(F_{2,135}=0.30, P>0.05\right)$, but significant species $\times$ site interaction indicated differences at some sites; concentrations were generally greater in hog sucker than in sunfish or stoneroller. Within species, differences among sites were highly significant $(P<0.01)$ in stoneroller but less so $(P<0.05)$ in hog sucker and sunfish (Table 3). In hog sucker, concentrations were significantly greater at Site 4 than at all others, largely as a consequence of the one large value noted previously. Concentrations in sunfish were higher on average at Site 9 (Middle Fork at Black, a downstream site) than at all sites $<10 \mathrm{~km}$ downstream from mines, and were lowest in fish from Site 16 (Big River; Table 3). Liver $\mathrm{Zn}$ concentrations in sunfish from Sites 6 and 14 were relatively high (Table 3 ) despite the fish having been collected several kilometers downstream of the stonerollers. Among groups of sites, liver $\mathrm{Zn}$ concentrations were significantly $(P<0.01)$ lower in sunfish from the Big River than from all other groups (Table 3). Concentrations in stoneroller were significantly higher at Site 14 and several other sites near mines and at downstream sites than at reference sites and at Sites 15 (exploration area) and 16 (Big River), where concentrations were lowest. Concentrations at some sites near mines were also not significantly different from Site 14, however (Table 3). Some differences between groups of sites were also significant in stoneroller, but there was no clearly evident mining-related pattern to these differences.

Dry-weight blood and liver $\mathrm{Zn}$ concentrations were positively correlated in stoneroller but not in hog sucker or sunfish, and $\mathrm{Zn}$ concentrations in liver were generally slightly greater than those in blood in all species (Fig. 4). As was true for $\mathrm{Cd}$, the relationship in hog sucker was adversely affected by the one large liver $\mathrm{Zn}$ value representing the fish from Site 4; without this value the relationship would have been weakly positive (Fig. 4). Conversely, the relationship in sunfish was adversely affected by the low value representing the fish from Site 7. In addition, liver $\mathrm{Zn}$ concentrations were lower relative to blood $\mathrm{Zn}$ in five stoneroller from sites located $<10 \mathrm{~km}$ downstream of mines (Fig. 4).

\subsection{Cobalt and nickel in hog sucker}

Cobalt was detected in all blood and liver samples analyzed (nine of each). Concentrations in blood and liver were nearly identical (Fig. 5), ranging from $0.44 \mu \mathrm{g} / \mathrm{g} \mathrm{dw}$ in blood at Site 11 (Middle Fork near Redmondville) to $10.4 \mu \mathrm{g} / \mathrm{g} \mathrm{dw}$ in liver at Site 12 (Strother Creek). Differences among sites were highly significant $(P<0.01)$ in both blood and liver; concentrations in fish from Site 12 were significantly greater than those from Sites 11 and 16 (BR; Fig. 5). Concentrations in blood, but not liver, were also significantly (but not substantially) greater at Site 16 than at Site 11 (Fig. 5).

Nickel was also detected in all 18 samples analyzed. Concentrations of $\mathrm{Ni}$ in blood were about twice those in liver $(0.10-2.71 \mu \mathrm{g} / \mathrm{g} \mathrm{dw}$ in liver, $0.20-6.30 \mu \mathrm{g} / \mathrm{g} \mathrm{dw}$ in 

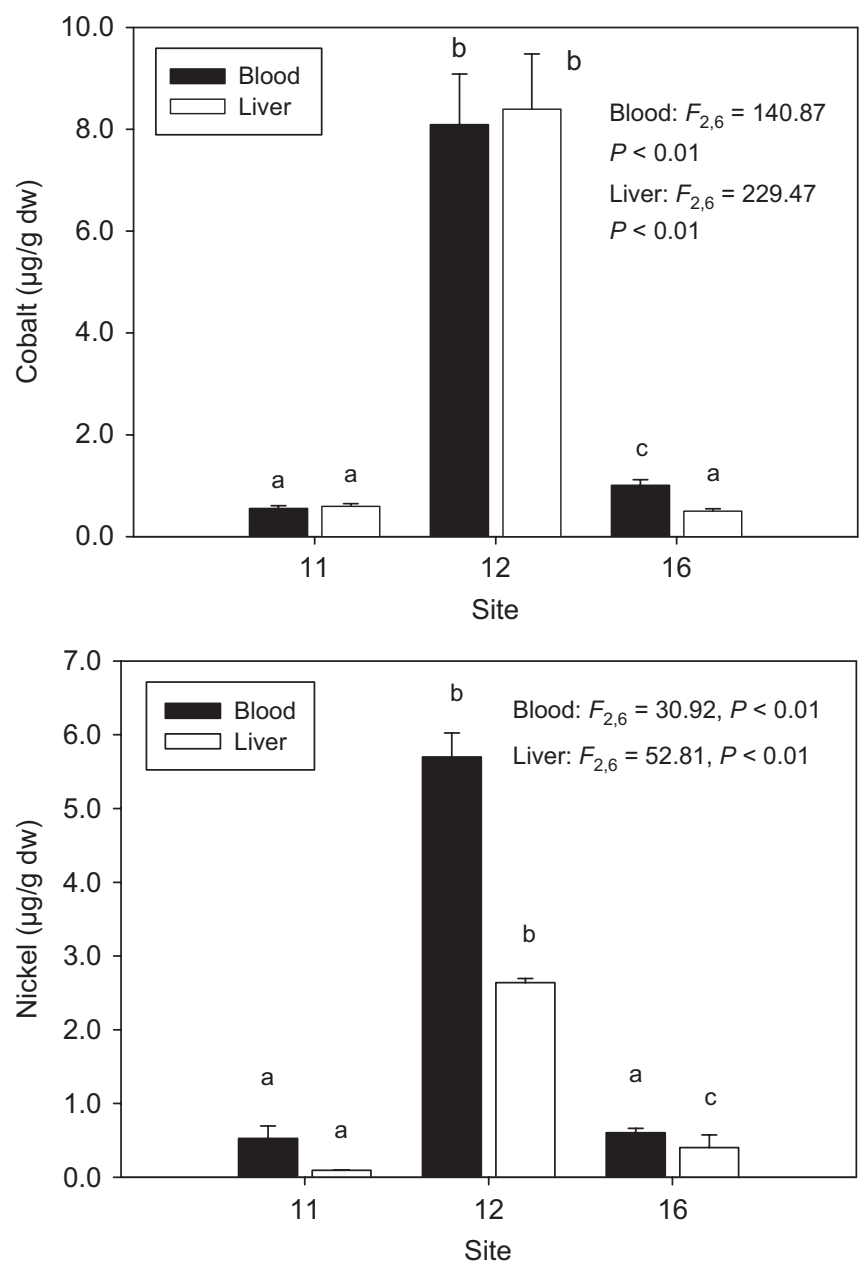

Fig. 5. Concentrations of cobalt and nickel in blood and liver of hog sucker from reference (Site 11) and mining-affected (Site 12) sites in the New Lead Belt and from the Big River (Site 16). Shown are arithmetic means and standard errors, and $F$-values and associated degrees-offreedom from analysis-of-variance. Within sample types (blood, liver), means containing the same letter are not significantly different $(P<0.05)$.

blood; Fig. 5). Differences among sites were significant $(P<0.01)$ in blood and liver and followed the same general pattern as $\mathrm{Co}$; both blood and liver concentrations were substantially higher at Site 12 than at either Sites 11 or 16 (Fig. 5). However, for Ni, concentrations in liver, but not blood, were also slightly (but nevertheless significantly) higher at Site 16 than at Site 11 (Fig. 5).

\section{Discussion}

Blood $\mathrm{Pb}$ concentrations in fish from mining-affected reaches of the Big River have not changed appreciably over two decades (Table 4). Mean concentrations of $5.00-13.4 \mu \mathrm{g} / \mathrm{g} \mathrm{dw}$ [estimated from wet-weight (ww) concentrations and $87.4 \%$ blood moisture] were reported in mixed sucker (Catostomidae, including hog sucker) from mining-affected sites upstream and downstream of Site 16 sampled in 1981; concentrations were $0.63 \mu \mathrm{g} / \mathrm{g} \mathrm{dw}$ at an upstream site (Table 4; Schmitt et al., 1984). Schmitt et al.
(1993) also reported a blood $\mathrm{Pb}$ mean of $19.1 \mu \mathrm{g} / \mathrm{g} \mathrm{dw}$ in black redhorse (Moxostoma duquesnii) collected from the Big River just upstream of Site 16 in 1989 (Table 4). These historical values bracket our Site 16 mean $(6.63 \mu \mathrm{g} / \mathrm{g} \mathrm{dw}$ for hog sucker; Tables 2 and 4) and are typical of blood $\mathrm{Pb}$ concentrations in catostomids from waters contaminated by mine tailings and smelters (Table 4; Schmitt et al., 1984, 1993, 2002). Mean blood $\mathrm{Pb}$ concentrations in sunfish obtained from contaminated parts of the Big River in 1980 were $3.41-7.22 \mu \mathrm{g} / \mathrm{g} \mathrm{dw}$ as compared to only $0.24 \mu \mathrm{g} / \mathrm{g} \mathrm{dw}$ at an upstream site (Table 4; Dwyer et al., 1988); our sunfish from Site 16 averaged $2.65 \mu \mathrm{g} / \mathrm{gdw}$ (Tables 2 and 4). Blood $\mathrm{Pb}$ concentrations were greater in largemouth and spotted bass (Micropterus spp.; 3.21-6.76 $\mu \mathrm{g} / \mathrm{g} \mathrm{dw}$ ) and common carp (Cyprinus carpio; $22.1 \mu \mathrm{g} / \mathrm{g} \mathrm{dw}$ ) collected at Site 16 concurrently with our fish (Brumbaugh et al., 2005) than in all of our samples (Fig. 2; Table 2). Conversely, blood $\mathrm{Pb}$ concentrations in stoneroller from Site 16 (mean $=1.39 \mu \mathrm{g} / \mathrm{g} \mathrm{dw}$ ) were only about $50 \%$ of those in sunfish and were less than all previously reported concentrations in fish from contaminated reaches of the Big River (Table 4).

Blood $\mathrm{Pb}$ concentrations in hog sucker from the Big River were about twice those from our sites near NLB mines, which were in turn as much as 10 -fold greater than reference and downstream sites (Tables 2 and 4). Blood $\mathrm{Pb}$ concentrations in several catostomids from sites near mines and smelters sampled by Schmitt et al. $(1993,2002)$ were also elevated relative to reference sites (Table 4); mean 1989 concentrations in hog sucker were $1.19 \mu \mathrm{g} / \mathrm{g} \mathrm{dw}$ at Site 14 (Courtois Creek) and $5.95 \mu \mathrm{g} / \mathrm{g} \mathrm{dw}$ at Site 2 (West Fork at West Fork). We did not obtain hog sucker from either of these sites in 2001, but mean concentrations in our fish from Sites 4 (Bee Fork), 7 (Sweetwater Creek), and 12 (Strother Creek) were within this range and were also $\leq 50 \%$ of concentrations in hog sucker from Site 16 (BR; Table 2). Mean blood $\mathrm{Pb}$ concentrations in hog sucker obtained in 1989 by Schmitt et al. (1993) from reference sites and sites further downstream of NLB mines were universally $<0.5 \mu \mathrm{g} / \mathrm{g} \mathrm{dw}$, as were ours (Tables 2 and 4 ).

In contrast to blood $\mathrm{Pb}$ concentrations, which were lower in the species we analyzed from the Big River than in the carp and bass analyzed by Brumbaugh et al. (2005), liver $\mathrm{Pb}$ concentrations in the carp $($ mean $=3.34 \mu \mathrm{g} / \mathrm{g} \mathrm{dw}$ ) were within the range of our data for all species (Fig. 2; Table 3). Our liver $\mathrm{Pb}$ concentrations in fish from the Big River were also similar to those in largescale sucker (Catostomus macrocheilus) from a smelter-contaminated site on the Columbia River near Northport, Washington (mean $=6.38 \mu \mathrm{g} / \mathrm{g} \mathrm{dw}$, estimated from ww concentrations and moisture) analyzed by Schmitt et al. (2002). Liver metals concentrations in hog sucker and sunfish obtained from sites in Missouri and Arkansas during 1993-97 were also measured as part of the US Geological Survey National Water Quality Assessment Program (NAWQA; Petersen et al., 1998). Liver Pb concentrations in NAWQA hog sucker from sites with no history of mining (personal 
Table 4

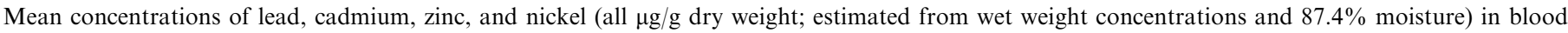
samples from suckers (Catostomidae) and longear sunfish collected from streams in southeastern Missouri by previous investigations, 1980-1993

\begin{tabular}{|c|c|c|c|c|c|c|}
\hline Taxon and location(s) & Year and [data source] & Nearest $\operatorname{site}(\mathrm{s})^{\mathrm{e}}$ & Lead & Cadmium & Zinc & Nickel \\
\hline \multicolumn{7}{|l|}{ Northern hog sucker ${ }^{\mathrm{a}}$} \\
\hline Big Creek near Hogan (R) & $1993[1]$ & ns & 1.03 & 0.24 & na & na \\
\hline Big Creek near Glover (M) & $1993[1]$ & ns & 9.44 & 1.43 & na & na \\
\hline Big Creek near Annapolis (M) & $1993[1]$ & ns & 4.92 & 1.06 & 47.6 & 0.82 \\
\hline Courtois Creek near Courtois (M) & 1989 [2] & 14 & 1.19 & 0.13 & 63.5 & 0.51 \\
\hline West Fork Black R. at West Fork (M) & $1989[2]$ & 2 & 5.95 & 0.05 & 56.3 & 0.76 \\
\hline West Fork Black R. near Centerville (D) & 1989 [2] & 1 & 1.43 & 0.04 & 62.7 & 1.06 \\
\hline Middle Fork Black R. near Edgehill (D) & 1989 [2] & 9 & 1.83 & 0.12 & 62.7 & 1.06 \\
\hline Huzzah Creek near Davisville (D) & 1989 [2] & 14 & 0.56 & 0.21 & 53.2 & 0.33 \\
\hline Hurricane Creek near Greer (R) & $1989[2]$ & 15 & $<4.29$ & 0.21 & 63.5 & 0.91 \\
\hline Reference sites $(\mathrm{R})$ & $2001[5]$ & 11 & 0.28 & 0.016 & 50.4 & 0.53 \\
\hline Mining sites $(\mathrm{M}, n=3)$ & $2001[5]$ & $4,7,12$ & $1.37-3.20$ & $0.033-0.745$ & $42.6-61.2$ & 5.70 \\
\hline Downstream sites (D, $n=2$ ) & $2001[5]$ & 8,13 & $0.20-0.36$ & $0.015-0.068$ & $44.0-48.0$ & na \\
\hline Big River below Desloge & $2001[5]$ & 16 & 6.63 & 0.075 & 48.4 & na \\
\hline \multicolumn{7}{|l|}{ Black or golden redhorse ${ }^{\mathrm{b}}$} \\
\hline Big R. near Desloge (M) & 1989 [2] & 16 & 19.13 & 0.07 & 72.2 & 0.71 \\
\hline Courtois Creek near Courtois (M) & 1989 [2] & 14 & 1.19 & 0.13 & 63.5 & 0.64 \\
\hline Indian Creek near Pea Ridge (M) & 1989 [2] & ns & 0.71 & 0.10 & 57.1 & 0.21 \\
\hline Logan Creek near Ellington (D) & 1989 [2] & 5 & 1.59 & 0.10 & 73.8 & 0.58 \\
\hline West Fork Black R. at Centerville (D) & 1989 [2] & 1 & 0.95 & 0.06 & 81.0 & 0.24 \\
\hline Middle Fork Black R. near Edgehill (D) & 1989 [2] & 9 & 0.95 & 0.06 & 87.3 & 1.09 \\
\hline Big Spring at Van Buren $(\mathrm{R}, n=2)$ & $1989[2]$ & ns & $<0.56-1.43$ & $0.02-<0.04$ & $49.2-60.3$ & $<0.14-0.27$ \\
\hline \multicolumn{7}{|l|}{ Mixed sucker spp. ${ }^{\mathrm{c}}$} \\
\hline Big R. at Irondale (R) & $1981[3]$ & ns & 0.63 & nd & 69.0 & na \\
\hline Big R. below Desloge $(n=3)$ & $1981[3]$ & 16 & $5.00-14.37$ & nd & $86.0-106$ & na \\
\hline \multicolumn{7}{|l|}{ Longear sunfish ${ }^{\mathrm{d}}$} \\
\hline Big R. at Irondale (R) & $1980[4]$ & ns & 0.24 & na & na & na \\
\hline Big R. below Desloge $(n=3)$ & $1980[4]$ & 16 & $3.41-7.22$ & na & na & na \\
\hline Reference sites $(\mathrm{R}, n=4)$ & $2001[5]$ & $3,10,11,15$ & $0.09-0.38$ & $0.003-0.030$ & $44.5-59.6$ & na \\
\hline Mining sites $(\mathrm{M}, n=6)$ & $2001[5]$ & $2,4,6,7,12,14$ & $0.44-1.32$ & $0.005-0.042$ & $43.2-69.4$ & na \\
\hline Downstream sites (D, $n=5$ ) & $2001[5]$ & $1,5,8,9,13$ & $0.09-0.39$ & $0.002-0.033$ & $43.3-62.8$ & na \\
\hline Big R. below Desloge & $2001[5]$ & 16 & 2.65 & 0.029 & 51.1 & na \\
\hline
\end{tabular}

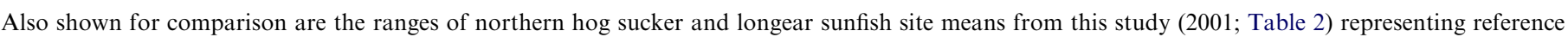

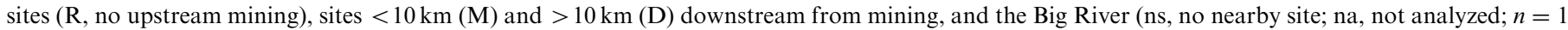

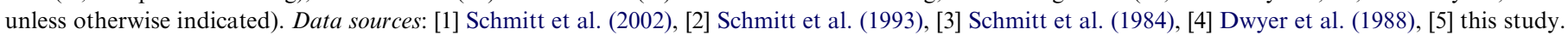

${ }^{\text {a } H y p e n t e l i u m ~ n i g r i c a n s . ~}$

${ }^{\mathrm{b}}$ Moxostoma duquesnii, M. erythrurum.

${ }^{\mathrm{c}} H$. nigricans, $M$. duquesnii, $M$. erythrurum.

${ }^{\mathrm{d}}$ Lepomis megalotis.

${ }^{\mathrm{e}}$ As identified in Table 1.

communication from J. Petersen, USGS, Little Rock, Arkansas) were universally $<0.84 \mu \mathrm{g} / \mathrm{g} \mathrm{dw}$, similar to our values for reference and downstream sites (Table 5). However, and in contrast to our findings of high liver $\mathrm{Pb}$ at Site 12, NAWQA hog sucker from a site on Strother Creek downstream of our Site 12 were only $0.70 \mu \mathrm{g} / \mathrm{g} \mathrm{dw}$, which were also within the range of out reference and downstream values (Table 5).

Among-site differences in blood and liver $\mathrm{Pb}$ concentrations were not as evident in stoneroller or sunfish as in hog sucker. Nevertheless, elevated blood and liver $\mathrm{Pb}$ concentrations at mining-affected sites (Big River and NLB) relative to reference and downstream sites were clearly evident in all three species (Fig. 2). Similarly, liver $\mathrm{Pb}$ concentrations in sunfish from southeastern Missouri NAWQA sites outside of the Black River watershed were all $<0.2 \mu \mathrm{g} / \mathrm{g} \mathrm{dw}$, but were higher in sunfish from several sites on the West Fork and Middle Fork near our Sites 1-4, 8 , and 9 (Table 5). The two largest of these concentrations, from Sites 2 (West Fork at West Fork) and 8 (Black River near Lesterville) exceeded all of our values for liver $\mathrm{Pb}$ in sunfish from the Big River as well as all sites near NLB mines (Tables 3 and 5). Liver $\mathrm{Pb}$ concentrations in shadow bass (Ambloplites ariommus) from the Eleven Point River downstream of our Site 15 and smallmouth bass (Micropterus dolomieu) from the West Fork at Greeley (our Site 3) obtained in 2001 by Femmer (2004) were $<0.2 \mu \mathrm{g} / \mathrm{g} \mathrm{dw}$, similar to most of our values for sunfish from reference sites (Tables 3 and 5).

Cadmium concentrations were also elevated at some sites near NLB mines relative to reference and downstream sites, but variability was high and trends were less evident 
Table 5

Concentrations of lead, cadmium, zinc, nickel, and cobalt (all $\mu \mathrm{g} / \mathrm{g}$ dry weight) in liver samples of northern hog sucker, longear sunfish, smallmouth bass, and shadow bass collected from streams in southeastern Missouri (MO), 1993-2001, by the US Geological Survey (USGS) ${ }^{\mathrm{a}}$

\begin{tabular}{|c|c|c|c|c|c|c|}
\hline Species and location(s) & Nearest site $(\mathrm{s})^{\mathrm{f}}$ & Lead & Cadmium & Zinc & Nickel & Cobalt \\
\hline \multicolumn{7}{|l|}{ Northern hog sucker ${ }^{\mathrm{b}}$} \\
\hline Yocum Creek near Oak Grove, AR $(\mathrm{R}, n=2)$ & ns & $<0.20-<0.84$ & $1.80-4.15$ & $114-131$ & $<0.20-<0.84$ & $0.40-<0.84$ \\
\hline Strother Creek near Redmondville, MO (M) & 12 & 0.70 & 1.40 & 145 & 1.20 & 3.80 \\
\hline Reference sites, this study $(\mathrm{R})$ & 11 & 0.70 & 1.77 & 116 & $0.09^{\mathrm{i}}$ & $0.59^{\mathrm{i}}$ \\
\hline Mining sites, this study $(\mathrm{M}, n=3)$ & $4,7,12$ & $1.53-9.82$ & $0.52-11.1$ & $138-733$ & $2.64^{\mathrm{i}}$ & $8.53^{\mathrm{i}}$ \\
\hline Downstream sites, this study $(\mathrm{D}, n=2)$ & 8,13 & $0.14-0.60$ & $1.41-4.30$ & $135-149$ & na & na \\
\hline Big River, this study & 16 & 4.70 & 2.34 & 74.8 & $0.40^{\mathrm{i}}$ & $0.50^{\mathrm{i}}$ \\
\hline \multicolumn{7}{|l|}{ Longear sunfish ${ }^{\mathrm{c}}$} \\
\hline Non-mining sites, southeastern $\mathrm{MO}(\mathrm{R}, n=3)^{\mathrm{d}}$ & 3,15 & $<0.20-<0.20$ & $0.40-2.20$ & $62.5-71.1$ & $<0.20-0.40$ & $0.60-1.10$ \\
\hline Non-mining sites, northern AR $(\mathrm{R}, n=4)^{\mathrm{e}}$ & ns & $1.00-2.20$ & $1.00-2.20$ & $78.1-106$ & $0.30-0.80$ & $0.80-2.30$ \\
\hline W. Fork Black R. at West Fork, MO (M) & 2 & 8.30 & 6.70 & 85.9 & 2.60 & 3.00 \\
\hline W. Fork Black R. near Centerville, MO (D) & 1 & 0.90 & 6.10 & 111 & 1.10 & 1.50 \\
\hline M. Fork Black R. near Lesterville, MO (D) & 9 & 5.90 & 3.70 & 78.2 & 0.70 & 1.00 \\
\hline Black R. near Lesterville, MO (D) & 1 & $<0.30$ & 2.40 & 77.2 & 0.40 & 0.90 \\
\hline Reference sites, this study $(\mathrm{R}, n=4)$ & $3,10,11,15$ & $0.03-0.39$ & $0.91-7.54$ & $83.9-105$ & na & na \\
\hline Mining sites, this study $(\mathrm{M}, n=6)$ & $2,4,6,7,12,14$ & $0.44-1.76$ & $1.97-9.22$ & $89.5-113$ & na & na \\
\hline Downstream sites, this study $(\mathrm{D}, n=5)$ & $1,5,8,9,13$ & $0.08-0.44$ & $1.31-2.56$ & $81.9-110$ & na & na \\
\hline Big River, this study & 16 & 2.01 & 10.89 & 65.0 & na & na \\
\hline \multicolumn{7}{|l|}{ Smallmouth bass ${ }^{\mathrm{g}}$} \\
\hline W. Fork Black R. near Greeley, MO (R) & 3 & $<0.2$ & 1.0 & 70.0 & $<0.20$ & 1.20 \\
\hline \multicolumn{7}{|l|}{ Shadow bass ${ }^{\mathrm{h}}$} \\
\hline Eleven Point R. near Turner's Mill, MO (R) & 15 & $<0.1$ & 0.5 & 91 & $<0.10$ & 1.10 \\
\hline
\end{tabular}

Also shown for comparison are the ranges of northern hog sucker and longear sunfish site means from this study (Table 3) representing reference sites (R, no upstream mining), sites $<10 \mathrm{~km}(\mathrm{M})$ and $>10 \mathrm{~km}$ (D) downstream from New Lead Belt mines, and the Big River (na, not analyzed; ns, no nearby site; $n=1$ unless otherwise indicated).

${ }^{a}$ USGS hog sucker and sunfish data summarized by Petersen et al. (1998), obtained online at http://infotrek.er.usgs.gov/servlet/page?_pageid= $543 \&$ dad $=$ portal30\&_schema $=$ PORTAL30; smallmouth bass and shadow bass data from Femmer (2004); all USGS data represent composite liver samples $(n=1)$ of $7-10$ adult fish collected and analyzed according to Crawford and Luoma (1993).

${ }^{\mathrm{b}}$ Hypentelium nigricans.

${ }^{\mathrm{c}}$ Lepomis megalotis.

${ }^{\mathrm{d} D o u s i n b u r y}$ Creek near Wall Street; Big Creek near Mauser Mill; Jacks Fork R. at Alley Spring.

${ }^{\mathrm{e}}$ Kings R. near Berryville; Buffalo R. near Boxley; N. Sylamore Creek near Fifty Six; Strawberry R. near Poughkeepsie.

${ }^{\mathrm{f}}$ As identified in Table 1.

${ }^{\mathrm{g}}$ Micropterus dolomieu.

${ }^{\mathrm{h}}$ Ambloplites ariommus.

i $n=1$.

than those for $\mathrm{Pb}$. Blood $\mathrm{Cd}$ concentrations in all species were greatest in fish from some sites nearest to NLB mines and in the Big River; however, concentrations were also comparatively high at some downstream sites, and there was considerable overlap within and among sites (Figs. 3 and 4; Table 2). Blood Cd concentrations in hog sucker from NLB streams sampled in 1989 by Schmitt et al. (1993) were $0.04-0.21 \mu \mathrm{g} / \mathrm{g} \mathrm{dw}$ (site means; estimated from wetweight concentrations and $87.4 \%$ blood moisture), which exceeded many of our site means (Tables 2 and 4) despite the fact that most of our NLB sites were closer to mines. Blood Cd in bass and carp obtained from the Big River concurrently with our fish averaged $0.125 \mu \mathrm{g} / \mathrm{g} \mathrm{dw}$ in carp and $<0.007-0.013 \mu \mathrm{g} / \mathrm{g} \mathrm{dw}$ in bass (Brumbaugh et al., 2005 ), about 2 -fold greater than mean concentrations in the species we analyzed (Table 2).

Spatial trends for liver $\mathrm{Cd}$ concentrations in hog sucker and stoneroller were similar to trends for blood $\mathrm{Cd}$, but concentrations in sunfish were highest at Site 16 (Big River;
Table 3). Liver Cd averaged $17.9 \mu \mathrm{g} / \mathrm{g} \mathrm{dw}$ in the carp from this site analyzed by Brumbaugh et al. (2005), which also exceeded most concentrations in the species we analyzed (Fig. 3; Table 3). Harrison and Klaverkamp (1990) reported a mean liver $\mathrm{Cd}$ concentration of $1.33 \mu \mathrm{g} / \mathrm{g} \mathrm{dw}$ (estimated from ww concentrations and $79 \%$ moisture) in white sucker (Catostomus commersonii) from lakes near the smelter at Flin Flon, Manitoba (Canada) and $0.14-0.24 \mu \mathrm{g} / \mathrm{g} \mathrm{dw}$ in more distant lakes. Liver $\mathrm{Cd}$ in hog sucker from most of our NLB sites exceeded the maximum concentration reported by Harrison and Klaverkamp, some by as much as 10-fold (Table 3). Liver $\mathrm{Cd}$ in hog sucker from Yocum Creek, AR analyzed by NAWQA were $1.80-4.15 \mu \mathrm{g} / \mathrm{g} \mathrm{dw}$ whereas in Strother Creek downstream of Site 12 they were $1.40 \mu \mathrm{g} / \mathrm{g} \mathrm{dw}$ (Table 5). Although the latter were similar to our Site 12 concentrations, the Arkansas values were also within our range for sites near mines (Tables 3 and 5) even though there is no mining in the Yocum Creek watershed. Liver $\mathrm{Cd}$ concentrations in NAWQA sunfish 
were $0.40-2.20 \mu \mathrm{g} / \mathrm{g} \mathrm{dw}$ at seven sites outside the Black River watershed but were $2.40-6.70 \mu \mathrm{g} / \mathrm{g} \mathrm{dw}$ at four sites within the Black River downstream of the NLB (Table 5). These Black River concentrations were greater than all of our concentrations in sunfish (Fig. 3; Table 3). Overall, blood and liver $\mathrm{Cd}$ in our samples tended to be greatest at Sites 7 (Sweetwater Creek) and 14 (Courtois Creek), which are near NLB mines (Table 1). Liver Cd concentrations in smallmouth bass obtained in 2001 from the West Fork at Greeley and in shadow bass from the Eleven Point River were $\leqslant 1.0 \mu \mathrm{g} / \mathrm{g} \mathrm{dw}$ (Femmer, 2004), which were lower than most of our liver $\mathrm{Cd}$ concentrations in sunfish from reference sites (including Sites 3 and 15; Tables 3 and 5). The notable exception was our Site 11 (Middle Fork near Redmondville), a reference site, where liver Cd concentrations sunfish were as high as most sites located near mines (Table 3).

Zinc trends were even less evident than those for $\mathrm{Cd}$. Zinc is an essential element, and internal concentrations are tightly regulated by fish (Bury et al., 2003). Consequently, blood and liver concentrations may not reflect environmental exposure to the same extent as those of other elements, and differences related to mining exposure may not be evident (Schmitt et al., 1993). Blood Zn differences among our sites were statistically significant in sunfish and stoneroller, but not in hog sucker (Table 2). However, even in sunfish and stoneroller the differences between the highest and lowest concentrations were $<2$-fold. Liver $\mathrm{Zn}$ concentrations were lowest at Site 16 (Big River) in all three species analyzed (Table 3), and with the exception of one hog sucker from Site 4, minimum and maximum concentrations also differed by $<2$-fold. Mean blood $\mathrm{Zn}$ concentrations in the carp and bass analyzed by Brumbaugh et al. (2005) were $60.2-79.0 \mu \mathrm{g} / \mathrm{g} \mathrm{dw}$, and liver $\mathrm{Zn}$ in carp averaged $665 \mu \mathrm{g} / \mathrm{gdw}$. The carp and bass blood concentrations were within the range of the means for the species we analyzed, but the liver $\mathrm{Zn}$ concentrations in carp were substantially greater (Table 2). Harrison and Klaverkamp (1990) reported mean liver $\mathrm{Zn}$ concentrations of $205 \mu \mathrm{g} / \mathrm{g} \mathrm{dw}$ (estimated from ww concentrations and moisture) in white sucker from lakes near the smelter at Flin Flon, Manitoba, but only $48.6-90.5 \mu \mathrm{g} / \mathrm{g} \mathrm{dw}$ in more distant lakes. Schmitt et al. (2002) reported mean liver $\mathrm{Zn}$ concentrations of $237-258 \mu \mathrm{g} / \mathrm{g} \mathrm{dw}$ (estimated from ww concentrations and moisture) in largescale sucker from both reference and mining-contaminated sites in Washington. Liver $\mathrm{Zn}$ concentrations in hog sucker analyzed by NAWQA were $114-131 \mu \mathrm{g} / \mathrm{g} \mathrm{dw}$ in Yocum Creek, AR and $145 \mu \mathrm{g} / \mathrm{gdw}$ in Strother Creek downstream of Site 12 (Table 5); both are within the range of most of our liver $\mathrm{Zn}$ data (Fig. 4; Tables 3, 5). Similarly, liver Zn concentrations in NAWQA sunfish were only slightly higher (mean = $88.1 \mu \mathrm{g} / \mathrm{g} \mathrm{dw}$ ) from four sites in the Black River watershed than at seven sites outside $($ mean $=70.1 \mu \mathrm{g} / \mathrm{gdw}$; Table 5), which are all within the range of our concentrations in sunfish (Fig. 4; Tables 3 and 5). Liver $\mathrm{Zn}$ concentrations in the shadow bass and smallmouth bass obtained from the Eleven Point River and the West Fork at Greeley, respectively, in 2001 by Femmer (2004) were $70-91 \mu \mathrm{g} / \mathrm{g} \mathrm{dw}$, which were also within the range of most of our sunfish concentrations (Table 5). All of our liver $\mathrm{Zn}$ concentrations in hog sucker were lower than the range reported by Schmitt et al. (2002) for catostomids from sites near smelters and the maxima reported by Harrison and Klaverkamp (1990), but many of our values exceeded reference concentrations of the latter study. Nevertheless, there was no pattern indicating that blood and liver $\mathrm{Zn}$ concentrations were higher at sites near mines than at downstream or reference sites in the species we analyzed (Fig. 4).

Concentrations of $\mathrm{Pb}$ and $\mathrm{Cd}$ in blood and liver were correlated in the species we investigated (Figs. 2 and 3), but the relations between the tissues were weaker than those reported for carp by Brumbaugh et al. (2005). This difference can be attributed to both the narrower range of concentrations in the smaller fishes we sampled (including numerous censored blood $\mathrm{Cd}$ values) and to greater variability associated with small sample mass. The lack of correlation between blood and liver $\mathrm{Zn}$ concentrations is consistent with the results of Brumbaugh et al. (2005), however, and is further evidence that $\mathrm{Zn}$ is tightly regulated by fish (Bury et al., 2003). The liver represents a storage site for some metals, and concentrations may therefore reflect the long-term exposure history of the organism whereas the half-life of metals in blood may be only days or weeks (Savory et al., 1987). Consequently, the comparatively weak correlations between blood and liver metals concentrations and comparatively larger variation in liver than in blood may reflect varying temporal exposure patterns, which would be more apparent at sites near active mining operations than at historical mining sites.

The ores of the Viburnum Trend are enriched with a variety of metals, but only $\mathrm{Pb}, \mathrm{Zn}$, and $\mathrm{Cu}$ are recovered commercially in substantial quantities (Wixson and Jennett, 1975; Wixson, 1978). Small amounts of Co are recovered incidentally from $\mathrm{Pb}-\mathrm{Zn}$ mining in the NLB (Shedd, 2005), and the facility on Strother Creek releases solid wastes containing $\mathrm{Co}$ and $\mathrm{Ni}$ to the environment (Doe Run Company, 2003). Elevated concentrations of Co and $\mathrm{Ni}$ in streams as a consequence of historical mining have been reported elsewhere in southeastern Missouri (Proctor and Sinha, 1978; Proctor, 1984), but evidence for elevated concentrations in the $\mathrm{Pb}-\mathrm{Zn}$ mining areas is limited. Proctor (1984) reported that waters of the Big River contain elevated $\mathrm{Co}$ and $\mathrm{Ni}$, but concentrations of both metals were low in our hog sucker from the Big River (Fig. 5). Proctor (1984) also reported that Ni concentrations were elevated relative to other sites in the Meramec River basin in attached algae from Courtois and Indian Creeks, near our Site 14. We have no Ni data for Site 14, but this site was sampled in 1989 by Schmitt et al. (1992); blood Ni concentrations in hog sucker and black redhorse were low (Table 4). The greatest blood $\mathrm{Ni}$ concentrations in 
catostomids from Missouri reported by Schmitt et al. (1992) were $1.31 \mu \mathrm{g} / \mathrm{g} \mathrm{dw}$ (site mean; estimated from ww concentrations and moisture content) in golden redhorse (Moxostoma erythrurum) from a tailings-contaminated stream in southwestern Missouri and $1.06 \mu \mathrm{g} / \mathrm{g} \mathrm{dw}$ in hog sucker from two NLB sites (Middle Fork upstream of Site 9, and West Fork downstream of Sites 1-4; Table 4). Our blood Ni concentrations in hog sucker from Strother Creek were therefore at least 6-fold greater than any previously reported values from Missouri (Table 4). Blood Ni concentrations were only $0.71 \mu \mathrm{g} / \mathrm{g} \mathrm{dw}$ in black redhorse collected in 1989 from the Big River (Schmitt et al., 1992), which is similar to our value for hog sucker from the Big River and a reference site (Table 4). However, liver Ni concentrations in several species of fish from lakes near the Ni smelter at Sudbury, Ontario (Canada) were as high as $51.0-81.0 \mu \mathrm{g} / \mathrm{g} \mathrm{dw}$ (estimated from ww concentrations and $79 \%$ moisture) in the studies reviewed by Eisler (1998), which is about 10 -fold greater than our concentrations in hog sucker from Strother Creek (Fig. 5). Liver Ni concentrations in hog sucker obtained from Yocum Creek, AR in $1993-97$ by NAWQA were $<0.84 \mu \mathrm{g} / \mathrm{g} \mathrm{dw}$, which is similar to our value for Sites 11 and 16, whereas in Strother Creek downstream of Site 12 it was $1.20 \mu \mathrm{g} / \mathrm{g} \mathrm{dw}$, which is substantially less than our Site 12 concentrations (Fig. 5; Table 5). Liver Ni concentrations in NAWQA sunfish were also only $0.40-1.10 \mu \mathrm{g} / \mathrm{g} \mathrm{dw}$ at four Black River sites and $<0.20-0.80 \mu \mathrm{g} / \mathrm{g} \mathrm{dw}$ outside of the Black River watershed; and liver $\mathrm{Ni}$ in shadow bass and smallmouth bass obtained in 2001 from the Eleven Point River and the West Fork at Greeley, respectively, by Femmer (2004) were $<0.2 \mu \mathrm{g} / \mathrm{g} \mathrm{dw}$ (Table 5).

Nickel is considered an essential element that is homeostatically regulated by higher vertebrates (Eisler, 1998). Our data indicate that $\mathrm{Ni}$ is not well regulated by fish, however. Nickel is toxic to fish embryos at water concentrations as low as $11 \mu \mathrm{g} / \mathrm{L}$ (Birge and Black, 1980). It can be accumulated directly from water by fish eggs (Dave and Xiu, 1991; Brix et al., 2004) and by fish via the gills, from which it diffuses into the blood plasma (Pane et al., 2004a, b; Brix et al., 2004). Some forms of Ni are also teratogenic and carcinogenic; although these effects have not been reported in fish, they have been documented in frog embryos as a result of waterborne exposure (see studies reviewed by Eisler, 1998). Much of the Ni in fish tissues is associated with blood plasma (Pane et al., 2004b), which is reflected in the 2-fold greater concentrations in our blood than liver samples (Fig. 5). Nickel toxicity can be additive with that of other metals present in streams draining the $\mathrm{NLB}$, including $\mathrm{Cd}, \mathrm{Cu}, \mathrm{Pb}$, and $\mathrm{Zn}$ (Enserink et al., 1991). In fish, $\mathrm{Ni}$ has been shown to induce hyperactivity, as does $\mathrm{Pb}$ (Weber et al., 1991; Shafiq-urRehman, 2003), and to stimulate hemoglobin synthesis and erythropoiesis (Alkahem, 1994). However, Ni and $\mathrm{Pb}$ can also inhibit heme synthesis (Maines, 1980; Joselow, 1980), and both $\mathrm{Ni}$ and cadmium can inhibit the induction of cytochrome P-450 enzymes in fish (Brüschweiler et al.,
1996). Together, the metals present in the NLB could profoundly influence heme metabolism and ultimately, through effects on cytochrome P-450, the toxicity of organic chemicals (Maines, 1980), including those used in metal beneficiation.

Cobalt is also an essential element (it is a component of vitamin $\mathrm{B}_{12}$ ) that can also be toxic to fish. Liver Co concentrations in NAWQA hog sucker from Strother Creek downstream of Site 12 were $3.80 \mu \mathrm{g} / \mathrm{g} \mathrm{dw}$, whereas those from Yocum Creek, AR were $<0.84 \mu \mathrm{g} / \mathrm{gdw}$ (Table 5). The Strother Creek value was about $50 \%$ of our Site 12 concentrations whereas the Yocum Creek concentrations were similar to those in our suckers from Sites 11 and 16 (Table 5). Liver Co concentrations in the shadow bass from the Eleven Point River and smallmouth bass from the West Fork at Greeley obtained in 2001 by Femmer (2004) contained only $1.1-1.2 \mu \mathrm{g} / \mathrm{g} \mathrm{dw}$, which is similar to our concentrations in hog sucker from Sites 11 and 16 and lower than those from Site 12 (Table 5). Hatchery-reared rainbow trout contained $0.304-0.720 \mu \mathrm{g} / \mathrm{g} \mathrm{dw}$ blood Co (estimated from ww concentrations and moisture) and $0.071-0.323 \mu \mathrm{g} / \mathrm{g} \mathrm{dw}$ liver Co (Harms and Kunze, 1977), which are within the range of our values for fish from the Big River and a reference site and substantially lower than those from Strother Creek (Table 5). However, in contrast to the nearly equal blood and liver Co concentrations in hog sucker from the three sites we sampled (Fig. 5), concentrations in rainbow trout were greater in blood than in liver (Harms and Kunze, 1977). These data indicate that $\mathrm{Co}$, like $\mathrm{Ni}$, is not well regulated by hog sucker. The mechanism of Co toxicity in fish is unknown, but in contrast to the additive toxicity of $\mathrm{Ni}$ and other metals, Co can act as an antagonist with respect to the toxicity of other metals (Marr et al., 1998). In warmblooded vertebrates, Co can also stimulate heme synthesis and affect cytochrome P-450 (e.g., Maines and Sinclair, 1977; Maines et al., 1976; Spaethe and Jollow, 1989; Taylor, 1990), but its effects on heme homeostasis in fish is unknown. Also in contrast to $\mathrm{Ni}$, for which there are Water Quality Criteria, Co concentrations are not presently regulated in US waters (US Environmental Protection Agency, 2004).

Ecological injury to fish as a consequence of exposure to metals from historical and active $\mathrm{Pb}-\mathrm{Zn}$ mining has been well documented. In Missouri, biochemical effects related to $\mathrm{Pb}$ exposure in fish have been reported in many species (Dwyer et al., 1988; Schmitt et al., 1984, 1993, 2002, 2005 , 2006). Effects on heme synthesis [specifically, the inhibition of the enzyme $\delta$-aminolevulic acid dehydratase (ALA-D; E.C. 4.2.1.24)], were reported previously in largemouth and spotted bass (Microperus spp.; Schmitt et al., 2005), sunfish (Dwyer et al., 1988), and in multiple species of suckers (Schmitt et al., 1984, 1993, 2002). Higher-level effects on Missouri fishes have also been documented, but not as thoroughly. In addition to ALA-D inhibition, diminished bone strength, which could lead to increased mortality and reduced growth, 
was reported in sunfish from tailings-contaminated reaches of the Big River (Dwyer et al., 1988). A depauperate fish community and reduced abundance of Neosho madtom (Noturus placidus), a Federally-listed species, were associated with contaminants from historical $\mathrm{Pb}-\mathrm{Zn}$ mining in southwestern Missouri (Wildhaber et al., 2000).

The effects of metals on fish resulting from historical $\mathrm{Pb}-\mathrm{Zn}$ mining have been investigated and reported more thoroughly and at more levels of biological organization elsewhere. For example, organism-, population-, and community-level effects were associated with elevated $\mathrm{Pb}$ concentrations and reduced ALA-D activity in two species of catfish (Pimelodidae) inhabiting a tailingscontaminated stream in Brazil (Moraes et al., 2003). In the United States, westslope cutthroat trout (Oncorhynchus clarki lewisi) fed benthic macroinvertebrates from metals-contaminated reaches of the Cour d'Alene River, Idaho (USA) contaminated by historical $\mathrm{Pb}$ mining were deemed less fit relative to trout fed commercial fish food or uncontaminated invertebrates; effects included reduced feeding activity, increased numbers of macrophage aggregates, degeneration of mucosal epithelium in the pyloric caecae, hepatic metallothionein induction, hyperplasia of kidney cells, and vacuolization of glial cells (Farag et al., 1999). Cutthroat trout avoided water contaminated with metals at concentrations representative of those in mining-contaminated reaches of the Cour d'Alene River (Woodward et al., 1997), to which the authors attributed reduced fish populations in affected waters. Similarly, reduced survival and growth, along with degenerative liver changes, were induced in rainbow trout (Oncorhynchus mykiss) fed contaminated invertebrates from the Clark Fork River, Montana (USA), where free-ranging brown trout (Salmo trutta) showed physiological evidence of metals exposure such as lipid peroxidation and copper-containing liver inclusions (Farag et al., 1995) and where the abundance of trout was believed to be limited by recruitment (Woodward et al., 1994). In related studies, brown trout and rainbow trout fed metals-contaminated invertebrates from the Clark Fork experienced reduced growth and survival (Woodward et al., 1995a), and brown trout avoided water contaminated at metals concentrations and ratios typical of the Clark Fork (Woodward et al., 1995b). Other effects in one or both trout species included constipation, gut impaction, lipid peroxidation, decreased zymogen production, and sloughing of intestinal mucosal epithelial cells (Woodward et al., 1995a). The authors of these investigations concluded that the reduced trout standing crops in contaminated reaches of the Clark Fork were due to chronic dietary metals exposure and avoidance of contaminated waters. And finally, salmonid populations were reduced in or extirpated from contaminated streams of the Boulder River watershed, Montana where increased mortality along with a variety of sublethal effects were attributed to metals from historical mining (Farag et al., 2003).
Although effects of metals from $\mathrm{Pb}-\mathrm{Zn}$ mining have been documented at many levels of biological organization, it is important to note that mining typically releases multiple contaminants that vary widely in both concentration and proportions among locations. This is clearly evident from our data (Tables 2 and 3), previous studies in Missouri (Tables 4 and 5), and elsewhere. Once the metals are released to aquatic ecosystems, fish exposure and effects are further determined by water chemistry, ecosystem dynamics, and internal processes, which are also variable. In addition to the release of metals, mining may cause additional chemical and physical changes in streams, such as reduced $\mathrm{pH}$, nutrient enrichment, and physical habitat alteration, which may also profoundly affect fish in many ways (Wildhaber et al., 2000). Consequently, it is often difficult to differentiate cumulative, higher-level effects such as reduced growth and recruitment caused by metals from those of other stressors, and more difficult still to generalize from site-to-site without accounting for differing metals ratios and other factors (e.g., Wildhaber et al., 2000). Biological effects of metals exposure in the fish we analyzed are reported in the companion paper (Schmitt et al., 2007), and the ecological effects of mining are the focus of ongoing studies (Imes, 2002).

\section{Conclusions}

Concentrations of $\mathrm{Pb}$ in blood and liver of stoneroller, sunfish, and hog sucker from sites $<10 \mathrm{~km}$ downstream of active mines and mills in southeastern Missouri and from the Big River were elevated relative to concentrations at sites further downstream and at reference sites. Concentrations in fish from the exploration area were similar to those from reference sites. These findings, which are consistent with those of previous studies, indicate that $\mathrm{Pb}$ is released to streams from active mining sites in the NLB, where it is available for uptake by aquatic organisms. Concentrations of $\mathrm{Pb}$ were typically greatest in hog sucker, intermediate in sunfish, and lowest in stoneroller. Consistent with previous findings, and with some exceptions, blood $\mathrm{Pb}$ concentrations in NLB fish exceeded reference concentrations but were lower than those from the Big River and other historical mining areas in the US and elsewhere, as would be expected given the modern technology and environmental regulation of mining in the NLB.

Trends for $\mathrm{Cd}$ and $\mathrm{Zn}$ were less evident; although the highest concentrations of both metals were in fish of all species from sites $<10 \mathrm{~km}$ downstream from NLB mines, there was considerable variability within and among sites and species. Similar variability for $\mathrm{Cd}$ and $\mathrm{Zn}$ relative to $\mathrm{Pb}$ has been reported in fish from mining areas in Oklahoma, which may reflect differing long-term accumulation patterns for these metals (Brumbaugh et al., 2005).

Overall, our findings indicate that current metals concentrations in the exploration area are comparable to those in other areas of southern Missouri not affected by mining, as also reported by Femmer (2004). Concentrations 
of $\mathrm{Ni}$ and $\mathrm{Co}$ were elevated relative to a reference site and the Big River in hog sucker from one NLB site, but only three sites were investigated for these metals. Previous studies in the NLB (i.e., Wixson, 1978; Schmitt et al., 1992, 1993; Petersen et al., 1998; Femmer, 2004) had not reported elevated $\mathrm{Ni}$ or Co concentrations, which were noted during the course of our investigations (Fig. 5; Besser et al., 2003). Ongoing studies in the NLB are therefore investigating $\mathrm{Ni}$ and $\mathrm{Co}$ in addition to $\mathrm{Pb}, \mathrm{Zn}$, and $\mathrm{Cd}$ at a wider range of sites, and are seeking to determine whether ecological injury associated with exposure to these metals has occurred.

\section{Acknowledgments}

J. Whyte, J.A. Allert, D. Nicks, J. Candrl, M. Annis, R. Lipkin, A. Donahue, J. Arms, M. Walther, R. Wiedemeyer, D. Hardesty, D. Whites, S. Olson, D. Parris, and J. Besser, all of our laboratory (CERC), participated in the collection and processing of the fish. P. Ciesclewicz and M. Reed of the Missouri Department of Conservation also assisted with fish collections. M. Walther, J. Arms, and S. Koppi of CERC assisted with metals analyses. J. Petersen of USGS provided the NAWQA data. A.L. Allert, J. Besser, J. Fairchild, and J. Peterson (all USGS) and two anonymous reviewers provided useful comments that were incorporated into this paper. We greatly appreciate the help of the many private landowners who granted us permission to sample on their properties.

Disclaimer: This study was conducted by the Columbia Environmental Research Center (CERC) of the US Geological Survey (USGS) as part of a Congressionally funded investigation of the effects of mining in the Mark Twain National Forest of Missouri, USA. Fish were collected in accordance with a Wildlife Collectors Permit issued by the Missouri Department of Conservation. All procedures conformed to the "Guidelines for the Use of Fishes in Research" of the American Fisheries Society (AFS), Institute of Fishery Research Biologists (AIFRB), and American Society of Ichthyologists and Herpetologists (ASIH) Use of Fishes in Research Committee (AFS, AIFRB, and ASIH, 2004) and with all USGS and CERC guidelines for the humane treatment of test organisms during culture and experimentation. Use of trade names does not constitute endorsement.

\section{References}

Alkahem, H.F., 1994. The toxicity of nickel and the effects of sublethal levels on haematological parameters and behaviour of the fish, Oreochromis niloticus. J. Univ. Kuwait Sci. 21, 243-252.

American Fisheries Society (AFS), American Institute of Fishery Research Biologists (AIFRB), and American Society of Ichthyologists and Herpetologists (ASIH), 2004. Guidelines for the use of fishes in research. American Fisheries Society, Bethesda, MD. 〈http:// www.fisheries.org/html/Public_Affairs/Sound_Science/Guidelines2004. shtml >.
Besser, J.M., Brumbaugh, W.G., Brunson, E.O., Allert, A.L., Schmitt, C.J., 2003. Metal bioavailability in stream and reservoir sediments from stream and reservoir sites in the vicinity of lead mining areas in Missouri. 24th Annual Meeting of the Society of Environmental Toxicology and Chemistry, November 11-15, Austin, TX. 〈http:// abstracts.co.allenpress.com/pweb/setac2003/document/?ID $=30614\rangle$.

Birge, W.J., Black, J.A., 1980. Aquatic toxicology of nickel. In: Nriagu, J.O. (Ed.), Nickel in the Environment. Wiley, New York, pp. 349-366.

Brix, K.V., Keithly, J., DeForst, D.K., Laughlin, J., 2004. Acute and chronic toxicity of nickel to rainbow trout (Oncorhynchus mykiss). Environ. Toxicol. Chem. 23, 2221-2228.

Brumbaugh, W.G., Schmitt, C.J., May, T.W., 2005. Concentrations of cadmium, lead, and zinc in fish from mining-influenced waters of Northeastern Oklahoma: sampling of blood, carcass, and liver for aquatic biomonitoring. Arch. Environ. Contam. Toxicol. 49, 76-88.

Brüschweiler, B.J., Würgler, F.E., Fent, K., 1996. Inhibitory effects of heavy metals on cytochrome P450A1 induction in permanent fish hepatoma cells. Arch. Environ. Contam. Toxicol. 31, 475-482.

Bury, N.R., Walker, P.A., Glover, C.N., 2003. Nutritive metal uptake in teleost fish. J. Expr. Biol. 206, 11-23.

Crawford, J.K., Luoma, S.N., 1993. Guidelines for studies of contaminants in biological tissues for the National Water-Quality Assessment Program. US Geological Survey Open-File Report 92-494, 69p.

Dave, G., Xiu, R.Q., 1991. Toxicity of mercury, copper, nickel, lead, and cobalt to embryos and larva of zebrafish, Brachydanio rerio. Arch. Environ. Contam. Toxicol. 21, 126-134.

Doe Run Company, 2003. Primary Mining and Smelting Division, 2002 Annual Report to our community, St. Louis, MO. 〈http://www. doerun.com/uploadfile/2002_Primary.pdf $>$.

Dwyer, F.J., Schmitt, C.J., Finger, S.E., Mehrle, P.M., 1988. Biochemical changes in longear sunfish, Lepomis megalotis, associated with lead, cadmium and zinc from mine tailings. J. Fish. Biol. 33, 307-317.

Eisler, R., 1998. Nickel hazards to fish, wildlife, and invertebrates: a synoptic review. Contaminant Hazard Reviews Report No. 34, Biological Science Report USGS/BRD/BSR-1998-001. US Geological Survey, Laurel, MD.

Enserink, E.L., Maas-Diepveen, J.L., Van Leeuwen, C.J., 1991. Combined effects of metals: an ecotoxicological evaluation. Water Res 25, 679-687.

Farag, A.M., Skaar, B., Nimick, D.A., MacConnell, E., Hogstrand, C., 2003. Characterizing aquatic health using salmonid mortality, physiology, and biomass estimates in streams with elevated concentrations of arsenic, cadmium, copper, lead, and zinc in the Boulder River watershed, Montana. Trans. Am. Fish. Soc. 132, 450-467.

Farag, A.M., Stansbury, M.A., Hogstrand, C., MacConnell, E., Bergman, H.L., 1995. The physiological impairment of free-ranging brown trout exposed to metals in the Clark Fork River, Montana. Can. J. Fish. Aquat. Sci. 52, 2038-2050.

Farag, A.M., Woodward, D.F., Brumbaugh, W.G., Goldstein, J.N., MacConnell, E., Hogstrand, C., Barrows, F.T., 1999. Dietary effects of metals-contaminated invertebrates from the Coeur d'Alene River, Idaho, on cutthroat trout. Trans. Am. Fish. Soc. 128, 578-592.

Femmer, S.R., 2004. Background and comparison of water-quality, streambed-sediment, and biological characteristics of streams in the Viburnum Trend and the exploration study areas, southern Missouri, 1995 and 2001. Water-Resources Investigations Report 03-4285, US Geological Survey, Reston, VA.

Gale, N., Adams, C.D., Wixson, B.F., Loftin, K.A., Huang, Y.-W., 2004. Lead, zinc, copper, and cadmium in fish and sediments from the Big River and Flat River Creek of Missouri's Old Lead Belt. Environ. Geochem. Health 26, 37-49.

Harms, U., Kunze, J., 1977. Determination of cobalt in small samples of tissues and organs of the rainbow trout (Salmo gairdneri) by flameless atomic-absorption-spectroscopy. Z. Lebensm. Unters. Forsch. 164, 204-207.

Harrison, S.E., Klaverkamp, J.F., 1990. Metal contamination in liver and muscle of northern pike (Esox lucius) and white sucker (Catostomus 
commersoni) and in sediments from lakes near the smelter at Flin Flon, Manitoba. Environ. Toxicol. Chem. 9, 941-956.

Imes, J.L., 2002. Geohydrological and biological investigations associated with a new lead-zinc exploration area near Winona, Missouri, and the Viburnum Trend of southeastern Missouri. Fact Sheet FS 0005-02, US Geological Survey, Reston, VA.

Joselow, M.M., 1980. Blood zinc and lead poisoning. In: Nriagu, J.O. (Ed.), Zinc in the Environment, Part II, Health Effects. Wiley, New York, pp. 171-181.

Maines, M.D., 1980. Nickel alterations of heme biosynthesis and degradation: implications for the oxidative metabolism of drugs and carcinogens. In: Nriagu, J.O. (Ed.), Nickel in the Environment. Wiley, New York, pp. 547-583.

Maines, M.D., Sinclair, P., 1977. Cobalt regulation of heme synthesis and degradation in avian embryo liver cell culture. J. Biol. Chem. 10, 219-223.

Maines, M.D., Janousěk, V., Tomio, J.M., Kappas, A., 1976. Cobalt inhibition and induction of $\delta$-aminolevulinate synthase in liver. Proc. Natl. Acad. Sci. USA 73, 1499-1503.

Marr, J.C.A., Hansen, J.A., Meyer, J.S., Cacela, D., Podrabsky, T., Lipton, J., Bergman, H.L., 1998. Toxicity of cobalt and copper to rainbow trout: application of a mechanistic model for predicting survival. Aquatic Toxicol 43, 225-238.

Moraes, M., Gerhald, P., Andersson, L., Shimada, H., Sturve, J., Rauch, S., Molander, S., 2003. Establishing causality between exposure to metals and effects on fish. Human Ecol. Risk Assess. 9, 149-169.

Pane, E.F., Haque, Z., Goss, G.G., Wood, C.M., 2004a. The physiological consequences of exposure to chronic, sublethal waterborne nickel in rainbow trout (Oncorhynchus mykiss): exercise vs resting physiology. J. Expr. Biol. 207, 1249-1261.

Pane, E.F., Haque, Z., Goss, G.G., Wood, C.M., 2004b. Mechanistic analysis of acute, Ni-induced respiratory toxicity in the rainbow trout (Oncorhynchus mykiss): an exclusively branchial phenomenon. Aquat. Toxicol. 69, 11-24.

Petersen, J., Adamski, J.C., Bell, R.W., Davis, J.V., Femmer, S.R., Freiwald, D.A., Joseph, R.L., 1998. Water quality in the Ozark Plateaus, Arkansas, Kansas, Missouri, and Oklahoma, 1992-1995. Circular 1158, US Geological Survey, Reston, VA.

Proctor, P.D., 1984. Heavy metal additions to the environment near mines, mills, and smelters, Southeast Missouri. In: Nriagu, J.O. (Ed.), Environmental Impacts of Smelters. Wiley, New York, pp. 89-115.

Proctor, P.D., Sinha, B., 1978. Cobalt-nickel-cadmium mobilization, transportation and fixation in surface waters, stream sediments and selected aquatic life in the Fredericktown Co-Ni Metallogenic Province, Southeast Missouri. In: Hemphill, D.D. (Ed.), Proceedings of the 12th Symposium on Trace Substances in Environmental Health (vol. XII), June 6-8, Columbia, MO, pp. 119-128.

Savory, J., Bertholf, R.I., Wills, M.R., 1987. In: Selier, H.G., Sigel, H., Sigel, A. (Eds.), Handbook on Toxicity of Inorganic Compounds. Marcel Dekker, New York, pp. 29-31.

Schmitt, C.J., Dwyer, F.J., Finger, S.E., 1984. Bioavailability of $\mathrm{Pb}$ and $\mathrm{Zn}$ from mine tailings by erythrocyte $\delta$-aminolevulinic acid dehydratase (ALA-D) activity in suckers (Pisces: Catastomidae). Can. J. Fish. Aquat. Sci. 41, 1030-1040.

Schmitt, C.J., Wildhaber, M.L., Hunn, J.B., Nash, T., Tieger, M.N., Steadman, B.L., 1992. Lead in Missouri streams: monitoring pollution from mining with an assay for $\delta$-aminolevulinic acid dehydratase (ALA-D) in fish blood. US Fish and Wildlife Service, Columbia National Fisheries Research Laboratory, Project Completion Report to the US Fish and Wildlife Service, Columbia, Missouri Field Office.
Schmitt, C.J., Wildhaber, M.L., Hunn, J.B., Nash, T., Tieger, M.N., Steadman, B.L., 1993. Biomonitoring of lead-contaminated Missouri streams with an assay for erythrocyte $\delta$-aminolevulinic acid dehydratase (ALA-D) activity in fish blood. Arch. Environ. Contam. Toxicol. $25,464-475$.

Schmitt, C.J., Caldwell, C.A., Olsen, B., Serdar, D., Coffey, M., 2002. Inhibition of erythrocyte $\delta$-aminolevulinc acid dehydratase (ALAD) activity in fish from waters affected by smelters. Environ. Monit. Assess. 77, 99-119.

Schmitt, C.J., Whyte, J.J., Brumbaugh, W.G., Tillitt, D.E., 2005. Biochemical effects of lead, zinc, and cadmium in the Tri-States district of northeastern Oklahoma, USA. Environ. Toxicol. Chem. 24, 1483-1495.

Schmitt, C.J., Whyte, J.J., Annis, M.L., Roberts, A.P., Tillitt, D.E., 2007. Biomarkers of metals exposure in fish from lead-zinc mining areas of Missouri, USA. Ecotoxicol. Environ. Saf. (submitted).

Shafiq-ur-Rehman, 2003. Lead-exposed increase in movement behaviour and brain lipid peroxidation in fish. J. Environ. Sci. Health A 38, 631-643.

Shedd, K.B., 2005. Cobalt. In: Metals and minerals: US Geological Survey Minerals Yearbook 2003, vol. I. pp. 19.1-19.16.

Spaethe, S.M., Jollow, D.J., 1989. Effect of cobalt protoporphyrin on hepatic drug-metabolizing enzymes. Specificity for cytochrome P-450. Biochem. Pharmacol. 38, 2027-2038.

Taylor, A., 1990. Cobalt carcinogenesis. In: Foulkes, E.C. (Ed.), Biological Effects of Heavy Metals, Metal Carcinogenisis, Vol. II. CRC Press, Boca Raton, FL, pp. 159-172.

US Environmental Protection Agency, 2004. National Recommended Water Quality Criteria. Office of Water, Office of Science and Technology (4304T), Washington, DC.

Weber, D.N., Russo, A., Seale, D.B., R.E. Spieler, R.E., 1991. Waterborne lead affects feeding abilities and neurotransmitter levels of juvenile fathead minnow Pimephales promelas. Aquat. Toxicol. 21, $71-80$.

Wildhaber, M.L., Allert, A.L., Schmitt, C.J., Tabor, V.M., Mulhern, D., Powell, K.L., Sowa, S.P., 2000. Natural and anthropogenic influences on the distribution of the threatened Neosho madtom in a Midwestern warmwater stream. Trans. Am. Fish. Soc. 129, 243-261.

Wixson, B.G., 1978. Biogeochemical cycling of lead in the New Lead Belt of Missouri. In: Nriagu, J.O. (Ed.), The Biogeochemical Cycling of Lead in the Environment, Part A. Ecological Cycles. Elsevier/North Holland Biomedical Press, Amsterdam, pp. 119-136.

Wixson, B.G., Jennett, J.C., 1975. The new lead belt in the forested Ozarks of Missouri. Environ. Sci. Technol. 9, 1128-1133.

Woodward, D.F., Brumbaugh, W.G., DeLonay, A.J., Little, E.E., Smith, C.E., 1994. Effects on rainbow trout fry of a metals-contaminated diet of benthic invertebrates from the Clark Fork River, Montana. Trans. Am. Fish. Soc. 123, 51-62.

Woodward, D.F., Farag, A.M., Bergman, H.L., DeLonay, E.E., Smith, C.E., Barrows, F.T., 1995a. Metals-contaminated benthic invertebrates in the Clark Fork River, Montana: effects on age- 0 brown trout. Can. J. Fish. Aquat. Sci. 52, 1992-1994.

Woodward, D.F., Hansen, J.A., Bergman, H.L., Little, E.E., DeLonay, A.J., 1995b. Brown trout avoidance of metals in water characteristic of the Clark Fork River, Montana. Can. J. Fish. Aquat. Sci. 52, 2031-2037.

Woodward, D.F., Goldstein, J.N., Farag, A.M., 1997. Cutthroat trout avoidance of metals and conditions characteristic of a mining waste site: Couer d'Alene River, Idaho. Trans. Am. Fish. Soc. 126, $699-706$. 